\title{
An Adaptive Transmission Scheme for Amplify-and-Forward Relaying Networks
}

\author{
Diana Pamela Moya Osorio, Member, IEEE, Edgar Eduardo Benítez Olivo, Member, IEEE, \\ Hirley Alves, Member, IEEE, José Cândido Silveira Santos Filho, Member, IEEE, \\ and Matti Latva-aho, Senior Member, IEEE
}

\begin{abstract}
In this paper, an adaptive scheme for amplify-andforward relaying networks is proposed, which selects a certain transmission mode for each communication process. Depending on the instantaneous channel conditions, one of the following modes is selected: direct transmission with no cooperation, cooperative transmission with half-duplex relaying and maximalratio combining at the destination, or cooperative transmission with full-duplex relaying and maximal-ratio combining at the destination. A three-node network is considered, containing a singleantenna source, a two-antenna relay that is able to implement full-duplex communication, and a single-antenna destination. Energy normalization per block is assumed, so that in those modes using cooperation, the system's transmission power is shared between source and relay. The performance analysis is provided in terms of outage probability and energy efficiency. We derive a tight approximate expression in closed form for the outage probability and an approximate expression in integral form for the mean energy consumption. The results show that our scheme outperforms all of transmission modes separately in terms of outage probability, while being more energy efficient than the cooperative transmission modes. In addition, the asymptotic analysis proves that the proposed scheme achieves full diversity order equal to 2 , thus outperforming those schemes with direct transmission or full-duplex cooperation only.
\end{abstract}

Index Terms-Amplify-and-forward, adaptive transmission mode, direct link, energy efficiency, full duplex, half duplex, outage probability, relaying networks.

\section{INTRODUCTION}

$\mathbf{R}$ ECENT studies regarding the feasibility of fullduplex (FD) transmissions have generated great expectations, by the reason of having transceivers capable to transmit and receive information simultaneously, and at the

Manuscript received February 3, 2016; revised June 17, 2016 and August 28, 2016; accepted September 28, 2016. Date of publication October 10, 2016; date of current version January 13, 2017. This work was supported in part by the Brazilian Ministry of Education (CAPES), the Finnish Centre for International Mobility (CIMO), and the Tekes and Academy of Finland (AKA). The associate editor coordinating the review of this paper and approving it for publication was I. Krikidis.

D. P. Moya Osorio is with the Department of Electrical Engineering, Center of Exact Sciences and Technology, Federal University of São Carlos, São Carlos 13565-905, Brazil (e-mail: dianamoya@ufscar.br).

E. E. Benítez Olivo is with São Paulo State University (UNESP), São João da Boa Vista 13876-750, Brazil (e-mail: edgar.olivo@sjbv.unesp.br).

H. Alves and M. Latva-aho are with the Department of Communications Engineering, University of Oulu, 90014 Oulu, Finland (e-mail: halves@ee.oulu.fi; matla@ee.oulu.fi).

J. C. S. Santos Filho is with the Department of Communications, School of Electrical and Computer Engineering, University of Campinas, Campinas 13083-852, Brazil (e-mail: candido@decom.fee.unicamp.br).

Color versions of one or more of the figures in this paper are available online at http://ieeexplore.ieee.org.

Digital Object Identifier 10.1109/TCOMM.2016.2616136 same frequency band, thus offering a variety of new solutions for the increasing demands of emerging wireless systems [1]. In virtue of this, applications on cooperative networks can rely on the FD mode as an efficient approach to improve the attainable spectral efficiency, by avoiding the inherent need of two channel uses per end-to-end transmission of its widely analyzed counterpart, the half-duplex (HD) mode. The main challenge to be tackled for the implementation of the FD transceiver is the self interference caused by the signal leakage between the transmitter and the collocated receiver. In this sense, FD relays can outperform HD relays as long as the self interference remains low, as shown in [2]. Different schemes using multiple-input multiple-output (MIMO) relays have been proposed to assess the mitigation of the self interference. In [3], time-domain cancellation is discussed; a variation of the spatial-domain suppression, called null-space projection, is analyzed in [4]; while both techniques are investigated together in [5]. Reports in [6]-[8] have shown that, through the combined use of passive and active (analog and digital) cancellation techniques, the self interference can be reduced to strikingly low values. Indeed, [6] shows that an average cancellation of $74 \mathrm{~dB}$ leads to higher FD rates if compared with HD rates, by using active cancellation to attenuate the line-of-sight (LOS) component of the self interference [9]. In this sense, the last advances in full-duplex transmission have generated great expectations, mainly for the spectral efficiency advantages, not only in cooperative networks, but for bidirectional communications or backhauling and access links in emerging wireless communication technologies for $5 \mathrm{G}[10],[11]$.

However, due to practical limitations such as hardware imperfections at the transmitter and receiver (for instance, nonlinearities and quantization noise on the digital converters), there is still a gap between the remaining interference and the receiver noise floor, even after the application of different stages of passive and active cancellation [12]. This remaining interference is known as residual self-interference (RSI), and it prevents FD to achieve a signal-to-noise ratio (SNR) comparable to that of HD. Also, the RSI renders a zerodiversity behavior for FD schemes, that is, the slope of the outage or bit-error-rate (BER) curves at high SNR is nil [13]. Thereby, the RSI has been taken into consideration for the various analyses of FD networks. This is commonly modeled as a Rayleigh fading channel [14]-[17]. The effects of RSI can be further alleviated through the use of power allocation, as shown in [18]. Indeed, in [19], it is shown that FD is able to outperform HD under an optimal power allocation 
scheme for an amplify-and-forward (AF) FD relaying system, when RSI is considered. Additionally, [20] proposes a hybrid scheme that opportunistically switches between FD and HD modes, in combination with transmit power adaptation. In that work, expressions for the instantaneous and average spectral efficiency are obtained for the hybrid $\mathrm{FD} / \mathrm{HD}$ scheme by finding switching boundaries where a certain mode presents a better performance. There, the direct link is considered as interference, and it is revealed that a premature mode selection in an early design stage leads to inefficient spectrum utilization, as there exists a trade-off that favors alternately the modes during operation, specially when transmit power adaptation is used to alleviate the effects of RSI in FD mode. On the other hand, in [13], an hybrid FD/HD relaying is employed in an optimal relay selection context. In that work, the outage probability is evaluated in a scenario where the direct link is totally neglected. Then, the hybrid scheme proved to outperform relay selection schemes based on HD alone, and it also overcomes the zero diversity-order problem inherent of FD schemes. In this context, [21] also addressed a hybrid FD/HD scheme by analyzing an optimal transmission scheduling that optimizes the end-to-end throughput by controlling the duration of each transmission. Also, in [22], scheduling and resource allocation are tackled as an optimization problem for a hybrid scheme, which dynamically selects between $\mathrm{AF}$ and DF (decode-and-forward) relaying protocols with FD and HD relays in MIMO and orthogonal frequency division multiple access (OFDMA) systems.

On the other hand, in the analysis of cooperative relaying networks, particularly in full-duplex (FD) relaying, most works only consider the non-cooperative strategies, that is, dual- or multi-hop scenarios, when the direct link is either assumed to be a source of interference [20], [23] or fully ignored [13], [22]. Recently, some works have explored the benefits of using the direct link in FD systems, such as in [16] and [24]-[27], specially to overcome the zero-diversity problem inherent to FD. In [16] and [24], the direct link was allowed to convey useful information in DF-FD relay networks. In the former, the outage performance was evaluated under Nakagami- $m$ fading, while in the latter an incremental selective DF scheme was studied. In [25], the error-rate and diversity performances were evaluated for an AF-FD network under RSI, in which the direct link is considered as a diversity path by using a linear relaying protocol. In [26], the direct link was exploited to transmit information in an AF-FD relaying network, and an approximate closed-form expression for the outage performance was derived. In that work, it was shown that the use of the direct link along with diversity combining at the destination permits to improve the diversity order and reliability obtained by FD schemes. Finally, in [27], the impact of the direct link was studied for a DF-FD system in which the self- and cross-talk interferences can be completely suppressed. In that work, it was shown that the direct link plays a critical role in the performance of FD relaying.

In this paper, we combine the benefits of hybrid FD/HD schemes and the exploration of the direct link by investigating the outage performance and the energy efficiency of an

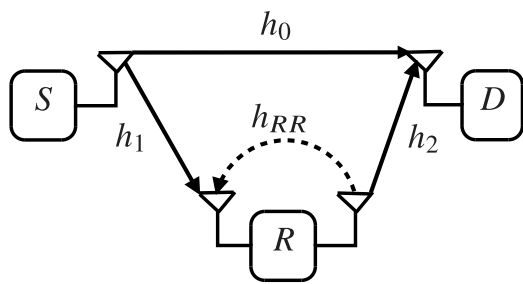

Fig. 1. System model composed by a source (S), a destination (D), and a FD relay (R) operating under AF protocol.

adaptive transmission-mode scheme (ATMS) for variable-gain $\mathrm{AF}$ relaying networks, where the direct link is used to convey information. This is in contrast to [13] and [20], in which the direct link is either fully ignored or treated as interference, respectively. In addition, differently from [20], we consider an instantaneous selection of the transmission mode at the source, instead of establishing switching boundaries. Also, differently from [13], the use of the direct link herein results in three (instead of two) distinct modes of transmission to be adaptively selected, namely: (i) direct transmission alone (no cooperation), (ii) cooperative transmission in HD mode by considering that the destination employs maximal-ratio combining (MRC) to merge the signals coming from the relay and the source, and (iii) cooperative transmission in FD mode along with MRC at the destination. This new scheme shall be investigated here in terms of its performance benchmark. The following are our main contributions:

- Approximate closed-form expressions are derived for the outage probability of the proposed ATMS.

- Asymptotic closed-form expressions are obtained for the outage probability of the proposed ATMS at high-SNR regime, indicating that it attains a full diversity order equal to 2 .

- Optimal power allocation and optimal relay position are analyzed through numerical evaluation.

- Approximate integral-form expressions for the mean energy consumption are derived, showing that the proposed ATMS outperforms HD+MRC and FD+MRC in terms of the energy efficiency.

The remainder of this paper is organized as follows. In Section II, the proposed mode-selection scheme is described. Section III investigates the outage probability by performing an approximate analysis, as well as an asymptotic characterization at high SNR. In Section IV, the mean energy efficiency of the proposed scheme is tackled. Section V shows some illustrative numerical results and draws some discussions. Finally, the main conclusions are summarized in Section VI.

Throughout this paper, $f_{Z}(\cdot)$ and $F_{Z}(\cdot)$ denote the probability density function (PDF) and the cumulative distribution function (CDF) of a generic random variable $Z$, respectively, $E\{\cdot\}$ denotes expectation, and $\operatorname{Pr}(\cdot)$ denotes probability.

\section{SYSTEM MODEL}

Fig. 1 depicts a two-hop relaying system composed by three nodes: one source $S$, one destination $D$, and one variable-gain AF-FD relay $R$. The source and destination are single-antenna devices, while the relay operates in FD mode 
by using one transmit antenna and one receive antenna. ${ }^{1}$ In this system, the signal belonging to the S-D link is not negligible, but used instead as an additional path to improve the reliability, as in [26]. In this context, we propose a scheme, ATMS, in which $S$ adaptively selects the transmission mode in order to optimize the performance of the system. Hence, based on the instantaneous channel state information (CSI), $S$ chooses one of the following options for transmission: (a) direct transmission with no cooperation (DT), (b) cooperative transmission in HD mode with MRC at $D$ (HD-MRC), and (c) cooperative transmission in FD mode with MRC at $D$ FD-MRC). Herein, we have considered that, for the HD-MRC mode, the relay uses a single antenna. However, as the relay already has two antennas to implement the FD-MRC mode, the HD-MRC mode can be, in principle, benefited in terms of performance by optimizing the two antenna configurations (e.g., placement and directivity) in two different directions [20]. Such a variant scheme shall not be treated in this work. Additionally, for energy saving purposes, we assume that a block energy normalization is executed by fixing as $P$ the total power transmitted per block. In virtue of this, for the DT mode, in which just direct transmission is considered, the source transmits with power $P$. Otherwise, for the HD-MRC and FD-MRC modes, in which relaying transmission is considered, the total power $P$ is distributed between $S$ and $R$ according to a power allocation factor $\eta$, defined as the ratio between the source transmission power and $P$. All the links in this network are subjected to independent block Rayleigh fading and additive white Gaussian noise with mean power $N_{0}$. Then, $W_{0}=\left|h_{0}\right|^{2} P_{S_{0}} / N_{0}, W_{1}=\left|h_{0}\right|^{2} P_{S_{1}} / N_{0}$, $X=\left|h_{1}\right|^{2} P_{S_{1}} / N_{0}$, and $Y=\left|h_{2}\right|^{2} P_{R} / N_{0}$ are the received SNRs of the direct link related to DT, direct link related to HD-MRC and FD-MRC, first-hop relaying link, and secondhop relaying link, respectively, in which (i) $h_{0}, h_{1}$, and $h_{2}$ are the corresponding channel coefficients, assumed to be independent circularly-symmetric Gaussian random variables $\mathcal{C N}\left(0, d_{i}^{-\beta}\right), i \in\{0,1,2\}$; (ii) $d_{0}, d_{1}$, and $d_{2}$ are the distances between the corresponding pairs of transceivers; (iii) $\beta$ is the pathloss exponent; and (iv) $P_{S_{0}}=P, P_{S_{1}}=\eta P$, and $P_{R}=(1-\eta) P$ are the transmission powers at $S$ and $R$ according to the selected mode of transmission, respectively. Furthermore, it is assumed that the RSI obtained after some stages of imperfect interference cancellation, when FD-MRC is selected for transmission, is modeled as a Rayleigh fading feedback channel with coefficient $h_{R R} \sim \mathcal{C N}\left(0, \sigma^{2}\right)$, with $U=\left|h_{R R}\right|^{2} P_{R} / N_{0}$ being the corresponding received SNR. Current designs to build in-band full-duplex radios, as those proposed by the research groups at Rice University [6] and Standford University [7], can achieve an interference mitigation of around $60 \mathrm{~dB}$ and $74 \mathrm{~dB}$, respectively, through the combination of passive and active (analog and digital) cancellation techniques, which is still far from the noise floor. However, after applying active cancellation, the Line-of-Sight (LoS) component of the residual self-interference is strongly reduced,

\footnotetext{
${ }^{1}$ It is worthwhile to mention that recent studies have reported systems implementing FD communication by using a single antenna as in [28]. However, this study focus on a two-antenna configuration at the relay.
}

so that the interference channel can be appropriately assumed as having a Rayleigh fading distribution [6], [14]-[17]. Besides, in this work we consider that the HD-MRC and FD-MRC modes operate as proposed in [26] and [29], respectively. In particular, for the HD-MRC mode, we consider that $S$ broadcasts information at the first time slot while $R$ and $D$ listen, and, at the second time slot, $R$ retransmits the information after applying an amplification gain as in [29, eq. (9)]. Then, $D$ performs a MRC of the signals coming from $S$ and $R$ at different time slots. On the other hand, for the FD-MRC mode, we assume that $S$ and $R$ transmit their signals, at the same time slot, separated by a processing delay greater than the symbol period, in order to guarantee that the relay receives and transmits uncorrelated symbols at a certain moment [20]. Then, it is also assumed that these signals are fully resolvable, co-phased, and merged via MRC at $D$. Further details on the operation of these modes and the relationships between transmitted and received signals can be found in [26] and [29]. ${ }^{2}$ Under these considerations, the endto-end SNRs for each mode are given by

$$
\begin{aligned}
\gamma_{\mathrm{DT}} & =W_{0} \\
\gamma_{\mathrm{HD}-\mathrm{MRC}} & =\frac{X Y}{X+Y+1}+W_{1} \\
\gamma_{\mathrm{FD}-\mathrm{MRC}} & =\frac{\frac{X}{U+1} Y}{\frac{X}{U+1}+Y+1}+W_{1} .
\end{aligned}
$$

\section{A. ATMS Process}

We assume that $S$ is responsible for coordinating the transmission-mode selection. Therefore, $S$ is able to acquire the global instantaneous CSI before the transmission takes place. In this context, we assume an ideal scenario in which the CSI is perfectly known by $S .^{3}$ In this way, we assume that $S$ continuously monitors the instantaneous CSI of the first- and second-hop relaying links as well as the direct link. Afterwards, $S$ selects the transmission mode that provides the maximum mutual information, that is,

$$
I_{\mathrm{ATMS}}=\max \left\{I_{\mathrm{DT}}, I_{\mathrm{HD}-\mathrm{MRC}}, I_{\mathrm{FD}-\mathrm{MRC}}\right\},
$$

where $I_{\mathrm{DT}}, I_{\mathrm{HD}-\mathrm{MRC}}$, and $I_{\mathrm{FD}-\mathrm{MRC}}$ are the mutual informations for the DT, HD-MRC, ${ }^{4}$ and FD-MRC modes, which can be respectively expressed as

$$
\begin{aligned}
I_{\mathrm{DT}} & =\log _{2}\left(1+W_{0}\right) \\
I_{\mathrm{HD}-\mathrm{MRC}} & =\frac{1}{2} \log _{2}\left(1+\gamma_{\mathrm{HD}-\mathrm{MRC}}\right) \\
& =\log _{2}\left(1+\sqrt{1+\gamma_{\mathrm{HD}-\mathrm{MRC}}}-1\right) \\
I_{\mathrm{FD}-\mathrm{MRC}} & =\log _{2}\left(1+\gamma_{\mathrm{FD}-\mathrm{MRC}}\right) .
\end{aligned}
$$

${ }^{2}$ It is worthwhile to mention that causality is maintained at $R$ for the FD-MRC mode as observed in [26, eq. (1)]

${ }^{3}$ This assumption is commonly used in benchmarking studies of related systems, (see, for instance, [13], [20]). In this way, such an ideal scenario offers a lower bound of the achievable outage performance for the proposed scheme.

${ }^{4}$ Observe that for the HD-MRC case a pre-log factor of $1 / 2$ appears due to the use of two time slots for the transmission of one block of information. 
Two stages are considered in order to accomplish the estimation of the corresponding CSI of all links at $S$. In a first stage, $S$ estimates the local CSI, that is, the CSI corresponding to the links $S-R$ and $S-D$, which can be achieved by using a pilot signaling coming from $R$ and $D$ as in [30], and by assuming channel reciprocity. By using a similar approach, in the second stage, $R$ can acquire its local CSI, that is, the CSI of the $R-D$ link and the self-interfering link. Subsequently, $R$ feeds back its local CSI to $S$, which concludes the transmission-mode selection. For that purpose, $R$ can use a quantized feedback similarly to that in $[31, \mathrm{Sec}$. VII], in which an overhead of $2 n$ bits is required, where $n$ corresponds to the number of quantization bits. For instance, by using $n=4,2^{4}=16$ uniform SNR quantization levels are adopted. Then, a feedback overhead of 8 bits is required for both links. Finally, $S$ broadcasts a 2-bit signaling message (e.g., " 00 ", " 01 ", or " 10 ", depending on which of the three transmission modes was selected) to inform $R$ and $D$ about the selected transmission mode.

\section{OUtage AnAlysis}

\section{A. Approximate Analysis}

In this section, the performance of the proposed scheme is characterized in terms of outage probability. By definition, the system is in outage when the instantaneous received SNR drops below a certain threshold $\tau \triangleq 2^{\mathcal{R}}-1$, where $\mathcal{R}$ is the target spectral efficiency given in bits/s/Hz. Herein, due to the intricate tractability for the exact analysis, we propose instead an approximate analysis by considering the wellknown approximation of the harmonic mean by the minimum as detailed in Appendix A. Hence, based on (4) and (5), the calculation of the approximate expression for the overall outage probability of the ATMS can be formulated as in the following theorem.
Theorem 1: The outage probability of ATMS can be expressed by the sum of three terms as

$$
P_{\mathrm{OUT}}=T_{1}+T_{2}+T_{3}
$$

where

$$
T_{1}=1-e^{-\frac{\eta \tau}{\bar{\gamma}_{0,1}}}+\frac{e^{-\left(\frac{\eta}{\bar{\gamma}_{0,1}}+\frac{1}{\bar{\gamma}_{2}}\right) \tau}\left(e^{\frac{\eta \tau}{\bar{\gamma}_{0,1}}}-e^{\left.\frac{\eta \tau}{\bar{\gamma}_{2}}\right) \bar{\gamma}_{2}}\right.}{\bar{\gamma}_{0,1}-\bar{\gamma}_{2}},
$$

and $T_{2}$ and $T_{3}$ can be approximated by the expressions in (8) and (9), respectively, at the bottom of this page. In these expressions, $\bar{\gamma}_{0,0}=E\left\{W_{0}\right\}, \bar{\gamma}_{0,1}=E\left\{W_{1}\right\}, \bar{\gamma}_{1}=E\{X\}$, $\bar{\gamma}_{2}=E\{Y\}, \bar{\gamma}_{R R}=E\{U\}$, and $\operatorname{Ei}(\cdot)$ is the exponential integral function as defined in [32, eq. (8.211)]. This result for the outage probability contains one single-fold integration in the term $T_{3}$.

Proof: See Appendix A.

Remark 1: A closed-form approximation for the integral in (9) can be obtained via the Gauss-Laguerre quadrature method [37, eq. (25.4.45)], which establishes that integrals of the form $\int_{0}^{\infty} e^{-x} f(x) d x$ can be approximated by a weighted sum of integrand samples as

$$
\int_{0}^{\infty} e^{-x} f(x) d x=\sum_{i=1}^{n} \omega_{i} f\left(l_{i}\right)+\epsilon,
$$

where $l_{i}$ is the $i$ th zero of the Laguerre polynomial $L_{n}(x)$ [37, eq. (22.2.13)], and $\omega_{i}=l_{i} /\left[(n+1) L_{n+1}\left(l_{i}\right)\right]^{2}$. To this end, we have performed the change of variables $w_{1}=\eta \tau\left(1-e^{-x}\right)$ and rewritten the integral accordingly. We have verified that a highly accurate approximation is attained, even for a small number of integrand samples, such

$$
\begin{aligned}
& T_{2} \approx-\frac{1}{\bar{\gamma}_{0,1} \bar{\gamma}_{R R}}\left(e^{\frac{\tau(\tau+1)}{\bar{\gamma}_{2}}}-1\right) e^{-\frac{\tau\left[\bar{\gamma}_{1}(\tau+2)+\bar{\gamma}_{2}\right]}{\bar{\gamma}_{1} \bar{\gamma}_{2}}}\left\{\frac{e^{-\tau\left(\frac{\eta}{\bar{\gamma}_{0,1}}-\frac{1}{\bar{\gamma}_{1}}\right)}\left(e^{\left.\frac{\eta \tau}{\bar{\gamma}_{0,1}}-e^{\frac{\eta \tau}{\bar{\gamma}_{2}}}\right) \bar{\gamma}_{0,1} \bar{\gamma}_{2} \bar{\gamma}_{R R}}\right.}{\bar{\gamma}_{0,1}-\bar{\gamma}_{2}}+\bar{\gamma}_{1} e^{\frac{\left[\bar{\gamma}_{0,1}\left(\bar{\gamma}_{1}+\bar{\gamma}_{2}\right)-\bar{\gamma}_{1} \bar{\gamma}_{2}\right]\left(\bar{\gamma}_{1}+\bar{\gamma}_{R R} \tau\right)}{\bar{\gamma}_{0,1} \bar{\gamma}_{1} \bar{\gamma}_{2} \bar{\gamma}_{R R}}}\right. \\
& \left.\times\left[\operatorname{Ei}\left(-\frac{\left[\bar{\gamma}_{0,1}\left(\bar{\gamma}_{1}+\bar{\gamma}_{2}\right)-\bar{\gamma}_{1} \bar{\gamma}_{2}\right]\left(\bar{\gamma}_{1}+\bar{\gamma}_{R R} \tau\right)}{\bar{\gamma}_{0,1} \bar{\gamma}_{1} \bar{\gamma}_{2} \bar{\gamma}_{R R}}\right)-\operatorname{Ei}\left(-\frac{\left[\bar{\gamma}_{0,1}\left(\bar{\gamma}_{1}+\bar{\gamma}_{2}\right)-\bar{\gamma}_{1} \bar{\gamma}_{2}\right]\left(\bar{\gamma}_{1}-(\eta-1) \bar{\gamma}_{R R} \tau\right)}{\bar{\gamma}_{0,1} \bar{\gamma}_{1} \bar{\gamma}_{2} \bar{\gamma}_{R R}}\right)\right]\right\} \\
& T_{3} \approx-\int_{0}^{\eta \tau} \frac{\bar{\gamma}_{R R}\left(\tau-w_{1}\right)}{\bar{\gamma}_{0,1}\left[\bar{\gamma}_{1}+\bar{\gamma}_{R R}\left(\tau-w_{1}\right)\right]} e^{\frac{\bar{\gamma}_{R R} w_{1}^{2}\left[\bar{\gamma}_{0,1}\left(\bar{\gamma}_{1}+\bar{\gamma}_{2}\right)-\bar{\gamma}_{1} \bar{\gamma}_{2}\right]-\bar{\gamma}_{R R} \tau w_{1}\left[\bar{\gamma}_{0,1}(\tau+3)\left(\bar{\gamma}_{1}+\bar{\gamma}_{2}\right)-\bar{\gamma}_{1} \bar{\gamma}_{2}\right]+\bar{\gamma}_{0,1} \tau\left\{\bar{\gamma}_{1}\left[\bar{\gamma}_{2}(\tau+1)+\bar{\gamma}_{R R} \tau(\tau+2)\right]+\bar{\gamma}_{2} \bar{\gamma}_{R R} \tau(\tau+2)\right\}}{\bar{\gamma}_{0,1} \bar{\gamma}_{1} \bar{\gamma}_{2} \bar{\gamma}_{R R}\left(w_{1}-\tau\right)} d w_{1}}
\end{aligned}
$$

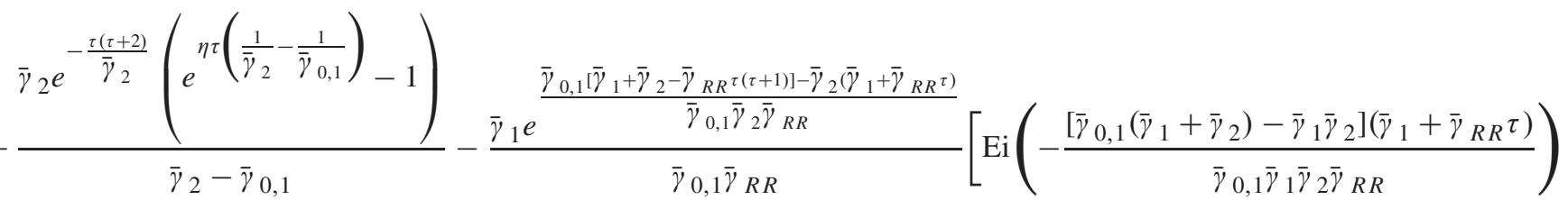

$$
\begin{aligned}
& \left.-\operatorname{Ei}\left(-\frac{\left[\bar{\gamma}_{0,1}\left(\bar{\gamma}_{1}+\bar{\gamma}_{2}\right)-\bar{\gamma}_{1} \bar{\gamma}_{2}\right]\left[\bar{\gamma}_{1}-(\eta-1) \bar{\gamma}_{R R} \tau\right]}{\bar{\gamma}_{0,1} \bar{\gamma}_{1} \bar{\gamma}_{2} \bar{\gamma}_{R R}}\right)\right]
\end{aligned}
$$


as $n=5$. Additionally, the truncation error can be obtained as $\epsilon=(n !)^{2} f^{(2 n)}(\xi) /(2 n)$ !, where $\xi$ lies somewhere in the range $0 \leq \xi<\infty$.

\section{B. Asymptotic Analysis}

In order to gain a better insight into the diversity order attained by the ATMS, its outage behavior at high SNR is formulated in the following theorem.

Theorem 2: The outage probability of the ATMS is asymptotically expressed as

$$
P_{\mathrm{OUT}}^{\infty}=T_{1}^{\infty}+T_{2}^{\infty}+T_{3}^{\infty},
$$

where

$$
\begin{aligned}
T_{1}^{\infty} & =\frac{\eta \tau^{2}(2-\eta)}{2 \bar{\gamma}_{0,1} \bar{\gamma}_{2}} \propto\left(\frac{1}{\bar{\gamma}}\right)^{2}, \\
T_{2}^{\infty} & =\frac{\tau(1+\tau)\left[\eta \tau \bar{\gamma}_{R R}-\bar{\gamma}_{1} \ln \left(\frac{\bar{\gamma}_{1}+\tau \bar{\gamma}_{R R}}{\bar{\gamma}_{1}+\tau \bar{\gamma}_{R R}(1-\eta)}\right)\right]}{\bar{\gamma}_{0,1} \bar{\gamma}_{2} \bar{\gamma}_{R R}} \\
& \propto\left(\frac{1}{\bar{\gamma}}\right)^{2}, \\
T_{3}^{\infty} & =-\frac{\eta \tau^{2}[\eta-2(2+\tau)]}{2 \bar{\gamma}_{0,1} \bar{\gamma}_{1}} \propto\left(\frac{1}{\bar{\gamma}}\right)^{2},
\end{aligned}
$$

with $\bar{\gamma} \triangleq 1 / N_{0}$ being defined as the system SNR.

Proof: See Appendix B.

Corollary 2.1: According to Theorem 2, as $T_{1}^{\infty} \propto(1 / \bar{\gamma})^{2}$, $T_{2}^{\infty} \propto(1 / \bar{\gamma})^{2}$, and $T_{3}^{\infty} \propto(1 / \bar{\gamma})^{2}$, then $P_{\text {OUT }}^{\infty} \propto(1 / \bar{\gamma})^{2}$. Consequently, the ATMS attains a full diversity order given as $\lim _{\bar{\gamma} \rightarrow \infty}-\frac{\log P_{\mathrm{OUT}}^{\infty}}{\log \bar{\gamma}}=2$.

Remark 2: Note that, due to the self interference, FD relaying schemes suffer from zero diversity order, which means that such relaying protocols achieve an outage floor at the highSNR regime [1], [2], [13]. One alternative to overcome this issue is by decoding the direct link together with the relaying link as reported in [26], which renders a diversity order of one. A higher diversity order of two can be achieved in our proposed scheme, as we allow the relay to opportunistically adapt its transmission mode, thus attaining the same diversity order of the HD-MRC mode. Nonetheless, our proposed scheme outperforms HD-MRC in terms of outage probability and throughput, as we shall see in Section V.

\section{ENERgy EFFICIENCY ANALYSIS}

In this section, we describe the proposed scheme in terms of energy efficiency. For this purpose, we refer to the work in [33], where an analysis of the total energy consumption per bit is performed for the incremental HD and selective DF-FD schemes. Accordingly, the total energy consumption per bit per Hertz is derived by considering the required power for transmission, the power consumed by the radiofrequency (RF) circuitry, and the target spectral efficiency. Besides, the baseband processing consumption is neglected, as it is much lower than in the RF circuitry. Bearing this in mind, we can express the total consumed mean energy in $\mathrm{J} / \mathrm{bit} / \mathrm{Hz}$ for the ATMS as

$\overline{\mathcal{E}}=\mathcal{P}_{\mathrm{DT}} \mathcal{E}_{\mathrm{DT}}+\mathcal{P}_{\mathrm{HD}-\mathrm{MRC}} \mathcal{E}_{\mathrm{HD}-\mathrm{MRC}}+\mathcal{P}_{\mathrm{FD}-\mathrm{MRC}} \mathcal{E}_{\mathrm{FD}-\mathrm{MRC}}$, where $\mathcal{E}_{\mathrm{DT}}, \mathcal{E}_{\mathrm{HD}-\mathrm{MRC}}$, and $\mathcal{E}_{\mathrm{FD}-\mathrm{MRC}}$ are the consumed energies per bit for each scheme independently, given respectively by ${ }^{5}$

$$
\begin{aligned}
\mathcal{E}_{\mathrm{DT}} & =\frac{1}{\mathcal{R}}\left(\frac{P_{S_{0}}}{\mu}+P_{\mathrm{TX}}+P_{\mathrm{RX}}\right) \\
\mathcal{E}_{\mathrm{HD}-\mathrm{MRC}} & =\frac{1}{\mathcal{R}}\left(\frac{P_{S_{1}}}{\mu}+\frac{P_{R}}{\mu}+2 P_{\mathrm{TX}}+3 P_{\mathrm{RX}}\right) \\
\mathcal{E}_{\mathrm{FD}-\mathrm{MRC}} & =\frac{1}{\mathcal{R}}\left(\frac{P_{S_{1}}}{\mu}+\frac{P_{R}}{\mu}+2 P_{\mathrm{TX}}+2 P_{\mathrm{RX}}\right),
\end{aligned}
$$

with $\mu$ being the drain efficiency of the amplifier, and $P_{\mathrm{TX}}$ and $P_{\mathrm{RX}}$ being the power consumed by the internal circuitry for transmitting and receiving, respectively. In this paper, as well as in [33], the reference values registered in [34] for $P_{\mathrm{TX}}$ and $P_{\mathrm{RX}}$ are considered. Therein, the circuit model for the transmitter considers the following blocks: digital-toanalog converter, mixer, transmit filters, and frequency synthesizer. On the other hand, the receiver circuitry considers the following blocks: frequency synthesizer, low-noise amplifier, mixer, intermediate frequency amplifier, receive filters, and analog-to-digital converter. Each block has its own power consumption values, which are available in [33]. Considering this, in this paper we assume the following values of the overall power consumption for transmitting and receiving: $P_{\mathrm{TX}}=97.9 \mathrm{~mW}, P_{\mathrm{RX}}=112.2 \mathrm{~mW}$, and $\mu=0.35$. Moreover, the consumed energy for each scheme is weighted by the probability of selecting each scheme at an instant, thus these probabilities are represented by the variables $\mathcal{P}_{\mathrm{DT}}, \mathcal{P}_{\mathrm{HD}-\mathrm{MRC}}$, and $\mathcal{P}_{\mathrm{FD}-\mathrm{MRC}}$, correspondingly. Approximate expressions for these probabilities can be calculated as shown in Theorem 3 .

Theorem 3: Single-fold integral-form approximate expressions for the probabilities of selecting DT, HD-MRC or FD-MRC for transmission in the ATMS are given as follows. In these, erf (.) stands for the error function [32, eq. (8.250-1)].

1) Direct Transmission Mode:

$$
\mathcal{P}_{\mathrm{DT}} \approx \mathcal{P}_{\mathrm{DT}}^{1}+\mathcal{P}_{\mathrm{DT}}^{2}+\mathcal{P}_{\mathrm{DT}}^{3}+\mathcal{P}_{\mathrm{DT}}^{4},
$$

with

$$
\begin{aligned}
& \mathcal{P}_{\mathrm{DT}}^{1}=1-\frac{\bar{\gamma}_{2}}{\bar{\gamma}_{0,0}-\eta \bar{\gamma}_{0,0}+\bar{\gamma}_{2}}, \\
& \mathcal{P}_{\mathrm{DT}}^{2}=\int_{0}^{\infty}\left(e^{\frac{w_{0}\left(1+w_{0}\right)}{\bar{\gamma}_{2}}}-1\right) e^{-w_{0}\left(\frac{1}{\bar{\gamma}_{0,0}}+\frac{1}{\bar{\gamma}_{1}}+\frac{2-\eta+w_{0}}{\bar{\gamma}_{2}}\right)}
\end{aligned}
$$

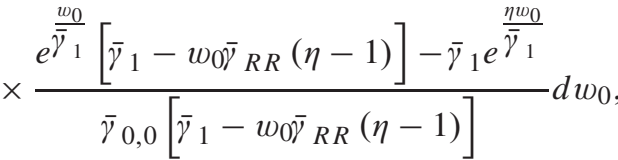

$$
\begin{aligned}
& \mathcal{P}_{\mathrm{DT}}^{3}=\int_{0}^{\infty} \frac{1}{\bar{\gamma}_{0,0}}\left(1-e^{\frac{1+w_{0}}{\bar{\gamma}_{R R}(\eta-1)}}\right) e^{-w_{0}\left(\frac{1}{\bar{\gamma}_{0,0}}+\frac{2-\eta+w_{0}}{\bar{\gamma}_{2}}\right)} \\
& +\bar{\gamma}_{1} e^{-\frac{\left.w_{0} \bar{\gamma}_{1} \bar{\gamma}_{2}+\bar{\gamma}_{0,0}\left[\bar{\gamma}_{1}\left(2-\eta+w_{0}\right)+\bar{\gamma}_{2}-\bar{\eta}_{2}\right]\right\}}{\bar{\gamma}_{0,0} \bar{\gamma}_{1} \bar{\gamma}_{2}}}
\end{aligned}
$$

\footnotetext{
${ }^{5}$ Herein, it is recognized that the HD-MRC requires to activate two times a transmission circuitry and three times a receiving circuitry, for the whole information transmission process, during the first and second time-slots. On the other hand, for the FD-MRC mode, it is required to activate only two times a transmission circuitry and two times a receiving circuitry [33].
} 


$$
\times \frac{e^{-\frac{\left(w_{0}+1\right)\left[w_{0} \bar{\gamma}_{R R}(\eta-1)-\bar{\gamma}_{1}\right]}{\bar{\gamma}_{1} \bar{\gamma}_{R R}(\eta-1)}}-1}{\bar{\gamma}_{0,0}\left[\bar{\gamma}_{1}-w_{0} \bar{\gamma}_{R R}(\eta-1)\right]} d w_{0},
$$

and $\mathcal{P}_{\mathrm{DT}}^{4}$ given as in (23), as shown at the bottom of this page.

2) Full-Duplex MRC Transmission Mode:

$$
\mathcal{P}_{\mathrm{FD}-\mathrm{MRC}} \approx \mathcal{P}_{\mathrm{FD}-\mathrm{MRC}}^{1}+\mathcal{P}_{\mathrm{FD}-\mathrm{MRC}}^{2}+\mathcal{P}_{\mathrm{FD}-\mathrm{MRC}}^{3},
$$

with

$$
\begin{aligned}
\mathcal{P}_{\mathrm{FD}-\mathrm{MRC}}^{1}= & \int_{0}^{\infty} \frac{\bar{\gamma}_{1}\left(e^{\frac{w_{0}\left(w_{0}+1\right)}{\bar{\gamma}_{2}}}-1\right)}{\bar{\gamma}_{0,0}\left[\bar{\gamma}_{1}-(\eta-1) \bar{\gamma}_{R R} w_{0}\right]} \\
& \times e^{-\frac{\left.w_{0}\left[\bar{\gamma}_{2} \bar{\gamma}_{0,0}-\bar{\gamma}_{0,0}+\bar{\gamma}_{1}\right)+\bar{\gamma}_{0,0} \bar{\gamma}_{1}\left(w_{0}-\eta+2\right)\right]}{\bar{\gamma}_{0,0} \bar{\gamma}_{1} \bar{\gamma}_{2}}} d w_{0}, \quad(25) \\
\mathcal{P}_{\mathrm{FD}-\mathrm{MRC}}^{2}= & \int_{0}^{\infty} \frac{e^{\left.\frac{w_{0}+1}{\left(\eta-1 \bar{\gamma}_{R R}\right.}-1\right)}}{\bar{\gamma}_{0,0} \frac{\left.w_{0}\left[\bar{\gamma}_{1} \bar{\gamma}_{2}+\bar{\gamma}_{0,0} \bar{\gamma}_{1}+\bar{\gamma}_{2}\right)\left(-\eta+w_{0}+2\right)\right]}{\bar{\gamma}_{0,0} \bar{\gamma}_{1} \bar{\gamma}_{2}}} \\
& -\frac{\bar{\gamma}_{1}\left(e^{-\frac{\left(w_{0}+1\right)\left((\eta-1) \bar{\gamma}_{R R} w_{0}-\bar{\gamma}_{1}\right)}{(\eta-1) \bar{\gamma}_{1} \bar{\gamma}_{R R}}}-1\right)}{\bar{\gamma}_{0,0}\left(\bar{\gamma}_{1}-(\eta-1) \bar{\gamma}_{R R} w_{0}\right)} \\
& \times e^{-\frac{\left.w_{0} \bar{\gamma}_{1} \bar{\gamma}_{2}+\bar{\gamma}_{0,0} \bar{\gamma}_{2}-\eta \bar{\gamma}_{2}+\bar{\gamma}_{1}\left(-\eta+w_{0}+2\right)\right]}{\bar{\gamma}_{0,0} \bar{\gamma}_{1} \bar{\gamma}_{2}}} d w_{0},
\end{aligned}
$$

and

$$
\begin{aligned}
\mathcal{P}_{\mathrm{FD}-\mathrm{MRC}}^{3}= & -\frac{\sqrt{\pi} \bar{\gamma}_{1} \bar{\gamma}_{2}}{2 \bar{\gamma}_{0,0} \sqrt{\bar{\gamma}_{1} \bar{\gamma}_{2}\left(\bar{\gamma}_{1}+\bar{\gamma}_{2}\right)}} e^{\frac{\left.\left[\bar{\gamma}_{1} \bar{\gamma}_{2}-(\eta-2) \bar{\gamma}_{0,0} \bar{\gamma}_{1}+\bar{\gamma}_{2}\right)\right]^{2}}{\left.4 \bar{\gamma}_{0,0} \bar{\gamma}_{1} \bar{\gamma}_{2} \bar{\gamma}_{1}+\bar{\gamma}_{2}\right)}} \\
& \times\left[\operatorname{erf}\left(\frac{\bar{\gamma}_{1} \bar{\gamma}_{2}-(\eta-2) \bar{\gamma}_{0,0}\left(\bar{\gamma}_{1}+\bar{\gamma}_{2}\right)}{2 \bar{\gamma}_{0,0} \sqrt{\bar{\gamma}_{1} \bar{\gamma}_{2}\left(\bar{\gamma}_{1}+\bar{\gamma}_{2}\right)}}\right)-1\right] .
\end{aligned}
$$

\section{3) Half-Duplex MRC Transmission Mode:}

$$
\mathcal{P}_{\mathrm{HD}-\mathrm{MRC}} \approx 1-\mathcal{P}_{\mathrm{DT}}-\mathcal{P}_{\mathrm{FD}-\mathrm{MRC}} .
$$

Proof: See Appendix C.

\section{Numerical Results AND Discussions}

In this section, we numerically evaluate our analytical results for some illustrative examples, and as check we contrast them to Monte Carlo simulations. For illustration purposes

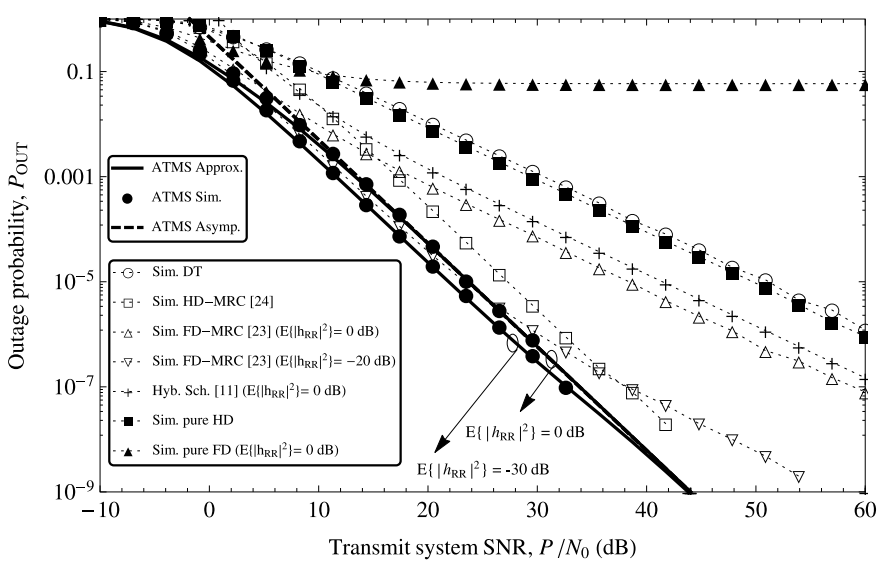

Fig. 2. Outage probability versus transmit system SNR for $\eta=0.5$.

and without loss of generality, we assume that the pathloss exponent is $\beta=4$ and the target spectral efficiency is $\mathcal{R}=1 \mathrm{bit} / \mathrm{s} / \mathrm{Hz}$. The sample network is generated in a linear topology, in which the distance between $S$ and $D$ is normalized to unity, i.e., $d_{0}=1$, which allows us to contrast the $\mathrm{S}-\mathrm{R}$ and R-D links to the S-D link in terms of average SNR, as in [26], [31], and [35].

Fig. 2 presents the outage probability of the ATMS as a function of the transmit SNR, $P / N_{0}$, for different values of RSI. Also, for comparison purposes, simulation results are depicted for the fixed modes: DT, FD-MRC [26] $\left(E\left\{\left|h_{R R}\right|^{2}\right\} \in\{0,-20\} \mathrm{dB}\right)$, HD-MRC [29], pure FD relaying for $E\left\{\left|h_{R R}\right|^{2}\right\}=0 \mathrm{~dB}$, and pure HD relaying. Additionally, we plot the outage probability of the hybrid scheme analyzed in [13, eqs. (59) and (68)] for $E\left\{\left|h_{R R}\right|^{2}\right\}=0 \mathrm{~dB}$, which neglects the direct link by selecting between pure FD and pure HD relaying. Notice that our approximation is very tight to the Monte Carlo simulations, specially at medium-to-high $\mathrm{SNR}$, as well as the corresponding asymptotes. It is observed that the ATMS offers an improvement in the performance if compared with any transmission mode individually, regardless of the level of RSI. Besides, the use of the direct link to convey information results in great benefits for the outage performance, in a way that the proposed ATMS proves to be significantly superior to the hybrid scheme in [13], as well as HD-MRC and FD-MRC prove superior to pure HD and pure FD relaying. Moreover, HD-MRC and FD-MRC relaying are superior even for the adaptive case in [13], which demonstrates the advantages of using the direct link. Importantly, note that the zero diversity order inherent of FD relaying is overcome by using the direct link, as is evidenced by comparing FD-MRC and pure FD. Additionally, it can be noticed that the

$$
\begin{aligned}
& P_{\mathrm{DT}}^{4}=\frac{\sqrt{\pi}}{2 \bar{\gamma}_{0,0}}\left\{\sqrt{\bar{\gamma}_{2}} e^{\frac{(\eta-1)^{2} \bar{\gamma}_{R R}^{2}\left[\bar{\gamma}_{2}-(\eta-2) \bar{\gamma}_{0,0}\right]^{2}+2(\eta-1) \bar{\gamma}_{0,0} \bar{\gamma}_{2} \bar{\gamma}_{R R}\left(\bar{\eta}_{0,0}-\bar{\gamma}_{2}\right)+\bar{\gamma}_{0,0}^{2} \bar{\gamma}_{2}^{2}}{4(\eta-1)^{2} \bar{\gamma}_{0,0}^{2} \bar{\gamma}_{2} \bar{\gamma}_{R R}^{2}}}\left[\operatorname{erf}\left(\frac{(\eta-1) \bar{\gamma}_{R R}\left[(\eta-2) \bar{\gamma}_{0,0}-\bar{\gamma}_{2}\right]+\bar{\gamma}_{0,0} \bar{\gamma}_{2}}{2(\eta-1) \bar{\gamma}_{0,0} \sqrt{\bar{\gamma}} \bar{\gamma}_{R R}}\right)+1\right]\right. \\
& \left.-\frac{\bar{\gamma}_{1} \bar{\gamma}_{2} e^{\frac{1}{(\eta-1) \bar{\gamma}_{R R}}}}{\sqrt{\bar{\gamma}_{1} \bar{\gamma}_{2}\left(\bar{\gamma}_{1}+\bar{\gamma}_{2}\right)}} e^{\frac{\left[(\eta-1) \bar{\gamma}_{R R}\left[(\eta-2) \bar{\gamma}_{0,0} \bar{\gamma}_{2}+\bar{\gamma}_{2}\right)-\bar{\gamma}_{1} \bar{\gamma}_{2}+\bar{\gamma}_{0,0} \bar{\gamma}_{1} \bar{\gamma}_{2}\right]^{2}}{\left.4(\eta-1)^{2} \bar{\gamma}_{0,0}^{2} \bar{\gamma}_{1} \bar{\gamma}_{2} \bar{\gamma}_{R R}^{2} \bar{\gamma}_{1}+\bar{\gamma}_{2}\right)}}\left[\operatorname{erf}\left(\frac{(\eta-1) \bar{\gamma}_{R R}\left[(\eta-2) \bar{\gamma}_{0,0}\left(\bar{\gamma}_{1}+\bar{\gamma}_{2}\right)-\bar{\gamma}_{1} \bar{\gamma}_{2}\right]+\bar{\gamma}_{0,0} \bar{\gamma}_{1} \bar{\gamma}_{2}}{2(\eta-1) \bar{\gamma}_{0,0} \bar{\gamma}_{R R} \sqrt{\bar{\gamma}_{1} \bar{\gamma}_{2}\left(\bar{\gamma}_{1}+\bar{\gamma}_{2}\right)}}\right)+1\right]\right\}
\end{aligned}
$$




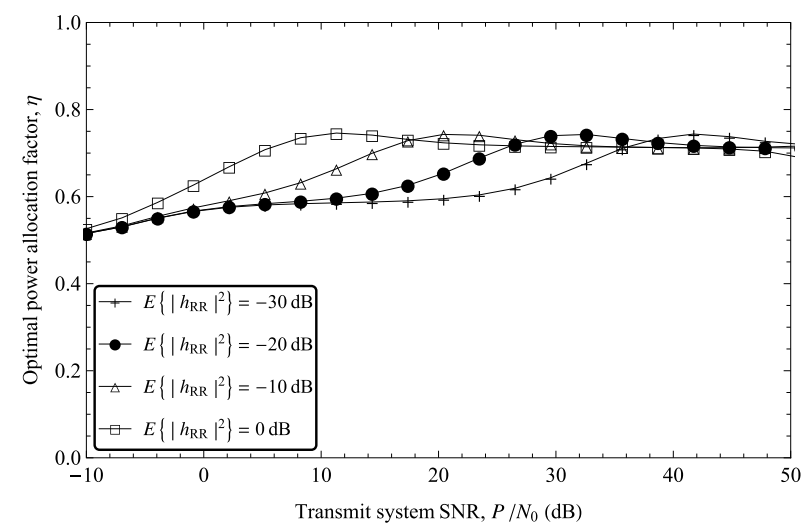

Fig. 3. Optimal $\eta$ versus transmit system SNR for different values of RSI.

ATMS is less susceptible to the impairments of the RSI, as the performance variation between the two different values of RSI analyzed is less significant if compared with the corresponding variation for FD-MRC. Moreover, at high SNR, the ATMS converges to the same values regardless of the level of RSI, which is corroborated by the matching of the asymptotes. This is because the ATMS opportunistically switches the transmission mode whenever the RSI compromises the rate. Moreover, the asymptotic curves corroborates that the ATMS attains full diversity order equal to that attained by HD-MRC, which is 2. Therefore, the ATMS outperforms the unitary diversity order attained by the FD-MRC [26].

Fig. 3 shows optimal values of the power allocation factor $\eta$ for the ATMS as a function of the transmit SNR. These values were obtained by a numerical solution of the following optimization problem:

$$
\eta_{\mathrm{opt}}=\arg \min _{\eta}\left\{P_{\mathrm{OUT}}(\eta)\right\} \quad \text { subject to } 0<\eta<1 \text {. }
$$

It is worth mentioning that (6) is a non-convex function and thus analytically finding the optimal allocation policy becomes a cumbersome task. Instead, we resort to a numerical optimization routine in Wolfram Mathematica called NArgMin [36], which always attempts to find a global optimum subject to the given constraints.

Results show that the optimal $\eta$ varies between $\sim 0.5$ and $\sim 0.7$, depending on the transmit SNR and on the level of RSI. The higher the RSI, the "faster" the optimal $\eta$ increases from $\sim 0.5$ to $\sim 0.7$ as the SNR increases. At high SNR, the optimal value tends to $\sim 0.7$. Therefore, as the RSI increases, less power should be allocated to the relay, as expected, in order to reduce the effects of the self interference. Furthermore, notice that even at high SNR the relay is still active, which is somewhat counter-intuitive, since all the transmission power could be in principle allocated to DT in such a case. Therefore, the proposed scheme favors cooperation without a significant change in the optimum power allocation policy, even at high SNR regime, since the destination is able to exploit not only the direct link but also the relaying link.

Figs. 4 and 5 show the optimal relay position as a function of the transmit SNR. In a manner similar to that of the optimization of $\eta$, the optimal relay position is obtained by

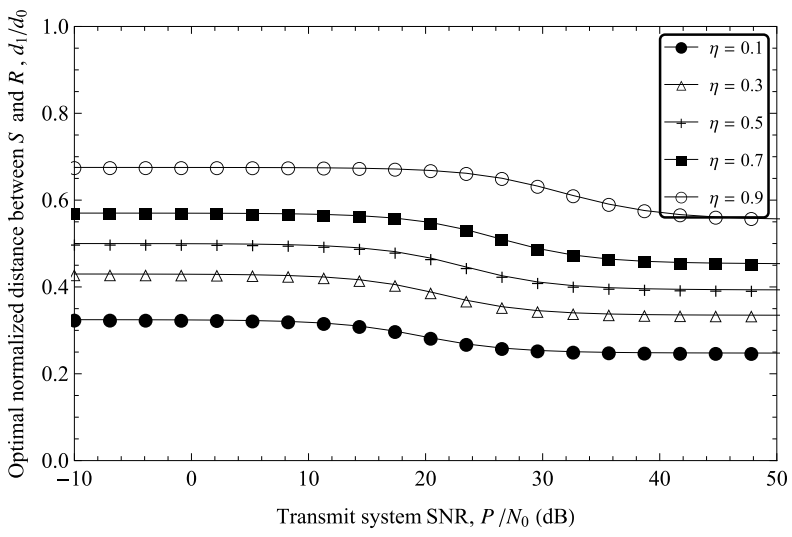

Fig. 4. Optimal distance between $S$ and $R$ versus transmit system SNR for different values of $\eta$ and $E\left\{\left|h_{R R}\right|^{2}\right\}=-20(\mathrm{~dB})$.

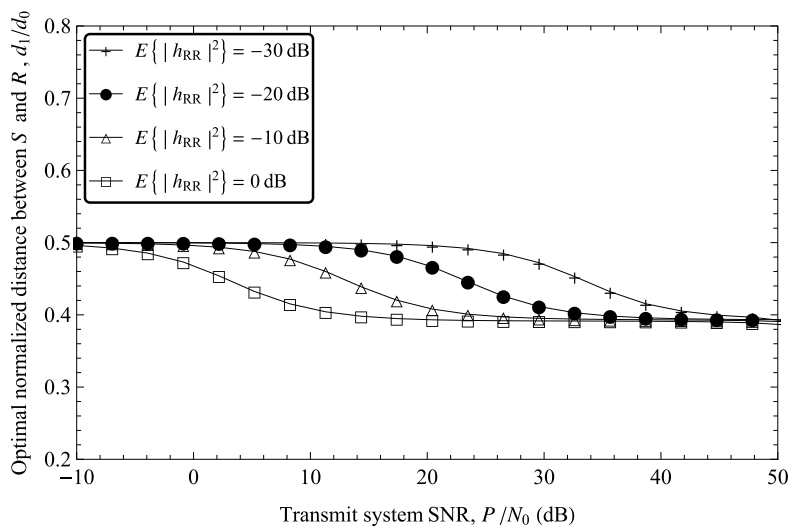

Fig. 5. Optimal distance between $S$ and $R$ versus transmit system SNR for different values of RSI and $\eta=0.5$.

evaluating numerically the following optimization problem:

$$
d_{1 \text { opt }}=\arg \min _{d_{1}}\left\{P_{\text {OUT }}\left(d_{1}\right)\right\} \text { subject to } 0<d_{1}<1 .
$$

Fig. 4 shows a comparison for different values of $\eta$. It is observed that the optimal relay position goes from $\sim 0.3$ to $\sim 0.7$ depending on $\eta$ and the SNR. The higher the value of $\eta$, the closer to $D$ is the optimal relay position. Besides, the higher the SNR, the closer to $S$ is the optimal relay position. On the other hand, Fig. 5 compares the optimal relay position for different levels of RSI when $\eta=0.5$. In this case, as the SNR increases, the optimal position tends to be nearer to $S$. Moreover, the higher the level of RSI, the faster the optimal position deviates toward $S$. From Figs. 4 and 5, it is also observed that the optimal normalized distance between $S$ and $R$ presents a floor at low and high SNR, which is independent on the level of RSI and varies according to the power allocation factor $\eta$. Indeed, this behavior obeys the balance condition of the first- and second-hop relaying links, which is a characteristic of the end-to-end SNR for the AF protocol. Thus, for the low SNR regime, where the effects of the RSI are smaller, the balance condition when the power is equally divided between $S$ and $R$ (i.e., for $\eta=0.5$ ) is attained in the middle of the way, that is $d_{1} / d_{0}=0.5$. However, for $\eta>0.5$, that is when more power is allocated to $S$, the relay approximates to $D$ in order to balance both links, and for the same reason, when $\eta<0.5$, the relay approximates to $S$. 


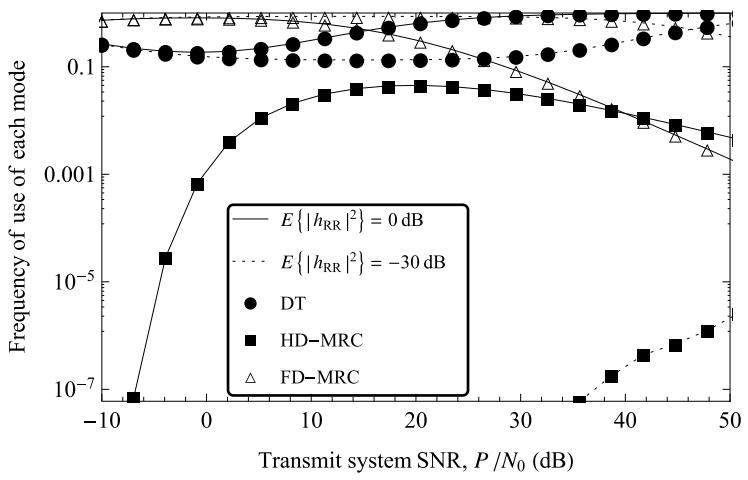

Fig. 6. Frequency of use for each transmission mode versus transmit system SNR for two different levels of RSI.

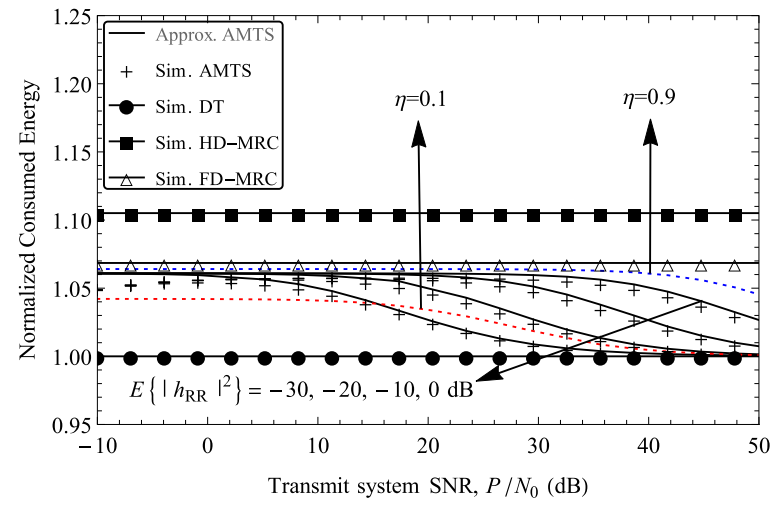

Fig. 7. Normalized energy consumption versus transmit system SNR for the ATMS with different values of RSI and $\eta=0.5$. Simulations of the special cases of DT, HD-MRC, FD-MRC and ATMS with $E\left\{\left|h_{R R}\right|^{2}\right\}=-20 \mathrm{~dB}$ for $\eta \in\{0.1,0.9\}$, are also shown in red and blue, respectively.

On the other hand, for the high SNR regime, it is observed that the optimal relay position slightly deviates toward $S$. This behavior can be explained by the fact that, when FD-MRC is the selected operation mode, the higher the system SNR, the higher the transmit power at the relay, and the worse the effects of the RSI. Then, the optimal position of R moves toward $\mathrm{S}$ in order to strengthen the first-hop relaying link, thus counterbalancing the effects of the RSI.

Fig. 6 shows simulations for the frequency of selection of each transmission mode in the ATMS as a function of the transmit SNR. It can be observed that the frequency of selection of HD-MRC is small if compared with DT and FDMRC, specially at lower levels of RSI. However, it is precisely this occasional selection of HD-MRC that enables ATMS to achieve full diversity equal to that attained by HD-MRC alone. Moreover, it can be noticed that FD-MRC is more frequently selected at low-to-medium SNR, while DT is more frequently selected at medium-to-high SNR. As discussed above, cooperation occurs even at high SNR, even though it is less frequent than DT. Under any scenario, the proposed ATMS approach alleviates the effects of fading and RSI by opportunistically switching among the transmission modes.

In Fig. 7 the normalized consumed energy $\overline{\mathcal{E}} / \mathcal{E}_{\mathrm{DT}}$ versus the transmit SNR is depicted for the ATMS with different levels of RSI and different values of $\eta$. Additionally, for comparison purposes, the energy consumption is plotted for each individual mode separately, as given in (16), (17), and (18).

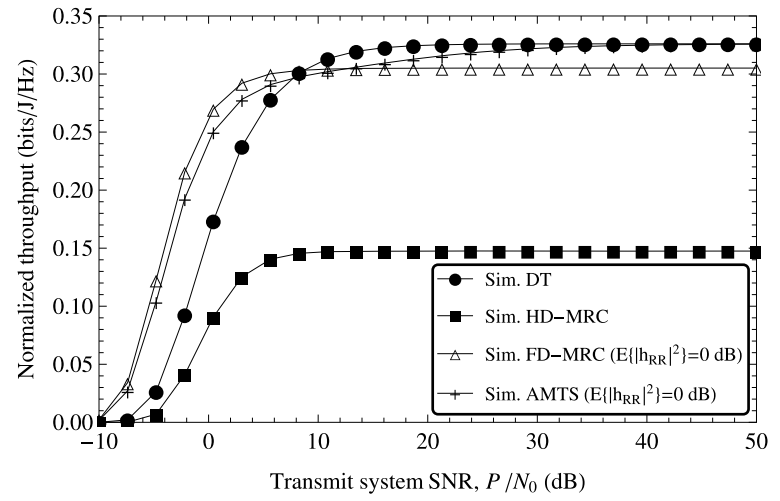

Fig. 8. Comparison of the normalized throughput versus transmit system SNR for the different modes of transmission and the ATMS.

We observe that the energy consumed by the ATMS is, in any case, somewhere between the energy consumed by DT and FD-MRC, even for different power allocations at $S$ and $R$. As expected, DT is the most energy efficient transmission mode, and HD-MRC is the least efficient. On the other hand, Fig. 8 shows the normalized system throughput (throughput/energy per bit per Hertz / target spectral efficiency) as a function of the transmit SNR for all transmission modes separately and the ATMS. Here, following [33] we define throughput as the spectral efficiency times the probability of successful transmissions, in bits/s/Hz. In this way, for a target spectral efficiency $\mathcal{R}$, the throughput is given by $T_{\mathrm{DT}}=$ $\mathcal{R}\left(1-P_{\mathrm{OUT}, \mathrm{DT}}\right)$ for $\mathrm{DT}$, by $T_{\mathrm{FD}-\mathrm{MRC}}=\mathcal{R}\left(1-P_{\mathrm{OUT}, \mathrm{FD}-\mathrm{MRC}}\right)$ for the FD-MRC, and $T_{\mathrm{HD}-\mathrm{MRC}}=0.5 \AA\left(1-P_{\mathrm{OUT}}\right.$,HD-MRC $)$ for HD-MRC. ${ }^{6}$ By normalizing the throughput by the energy consumed per bit per Hertz and by the target spectral efficiency, we have that the mean normalized throughput is given by

$$
\begin{aligned}
E\left\{\frac{T}{R \mathcal{E}}\right\}= & \frac{\left(1-P_{\mathrm{OUT}, \mathrm{DT}}\right) \mathcal{P}_{\mathrm{DT}}}{\mathcal{E}_{\mathrm{DT}}} \\
& +\frac{0.5\left(1-P_{\mathrm{OUT}, \mathrm{HD}-\mathrm{MRC}}\right) \mathcal{P}_{\mathrm{HD}-\mathrm{MRC}}}{\mathcal{E}_{\mathrm{HD}-\mathrm{MRC}}} \\
& +\frac{\left(1-P_{\mathrm{OUT}, \mathrm{FD}-\mathrm{MRC}}\right) \mathcal{P}_{\mathrm{FD}-\mathrm{MRC}}}{\mathcal{E}_{\mathrm{FD}-\mathrm{MRC}}} .
\end{aligned}
$$

The curves illustrated in Fig. 8 correspond to simulation results. We observe that the ATMS attains the best performance at high SNR among the cooperative schemes. At low SNR, the ATMS is very close to FD-MRC, but somewhat behind this. This difference at low-to-medium SNR regime can be explained by the fact that the ATMS opportunistically selects between the three modes, which present different throughput performances and energy requirements. Then, in terms of the throughput per energy consumption, the ATMS will not always be the best choice. However, the ATMS scheme is always very close to the best performance over the entire SNR range. As expected, the HD-MRC has the worst performance, in view of the need for two orthogonal channels for transmission. It is important to mention that, even though important gains can be attained in terms of throughput

\footnotetext{
${ }^{6}$ Notice that the 0.5 -factor in the HD-MRC case appears due to the requirement of two time slots per transmission. Bearing this in mind, $P_{\text {OUT }}$ is computed at different SNR thresholds: $\tau=2^{\mathcal{R}}-1$ and $\tau=2^{2 \mathcal{R}}-1$, for FD-MRC and HD-MRC, respectively.
} 
by the FD-MRC (duplicating the throughput of HD-MRC), an imperfect self-interference cancellation can deteriorate its outage performance in a way that can be less advantageous than HD-MRC. Therefore, a tradeoff between system throughput and outage performance is established. Considering this, the ATMS achieves a successful balance by alleviating the system susceptibility to the effects of residual self-interference approaching FD-MRC in terms of throughput.

\section{Vi. Conclusions}

We have proposed an adaptive scheme for amplify-andforward relaying networks that selects for each communication process a certain transmission mode among the following: direct transmission with no cooperation, cooperative transmission in HD mode with MRC at the destination, and cooperative transmission in FD mode with MRC at the destination. In our design we have considered an energy normalization per transmitted block, so that the modes using cooperation share the system's total power between $S$ and $R$. A numerical analysis of the optimal power allocation factor was presented. Also, a numerical analysis was developed to show the optimal position for $R$. A tight approximated closed-form expression for the outage probability was derived, and it was verified that our adaptive scheme outperforms whichever transmission mode operating separately, over the whole range of SNR. In addition, an approximate expression in integral form for the mean consumed energy of the proposed scheme was obtained, and the results show that our scheme is more energy efficient than the isolated modes using cooperative transmission. A throughput analysis per mean energy consumption confirmed the improved performance attained by the proposed adaptive scheme. Finally, an asymptotic analysis revealed that our adaptive scheme achieves full diversity order equal to 2 , thus outperforming the conventional schemes with direct transmission alone or full-duplex cooperation.

\section{APPENDIX A \\ APPROXimate CALCUlation FOR THE OUtAGE PROBABILITY OF THE ATMS}

The outage probability for the ATMS can be formulated as

$$
\begin{aligned}
& P_{\mathrm{OUT}}= \operatorname{Pr}\left(\max \left\{W_{0}, \sqrt{1+\gamma_{\mathrm{HD}-\mathrm{MRC}}}-1, \gamma_{\mathrm{FD}-\mathrm{MRC}}\right\}<\tau\right) \\
&= \operatorname{Pr}\left(W_{0}<\tau, \sqrt{1+\gamma_{\mathrm{HD}-\mathrm{MRC}}}-1<\tau, \gamma_{\mathrm{FD}-\mathrm{MRC}}<\tau\right) \\
&= \operatorname{Pr}\left(W_{1}<\eta \tau, \frac{X Y}{X+Y+1}<(\tau+1)^{2}-W_{1}-1,\right. \\
&\left.\quad \frac{\frac{X}{U+1} Y}{\frac{X}{U+1}+Y+1}<\tau-W_{1}\right) \\
& \stackrel{(a)}{=} T_{1}+T_{2}+T_{3},
\end{aligned}
$$

where (a) is obtained by splitting the analysis of the above probability into three terms according to the following ranges of values for the random variable Y: (i) $Y<\tau-W_{1}$, (ii) $\tau-$
$W_{1}<Y<(\tau+1)^{2}-W_{1}-1$, and (iii) $Y>(\tau+1)^{2}-W_{1}-1$. The term $T_{1}$ can be formulated as

$$
\begin{aligned}
T_{1} & =\operatorname{Pr}\left(Y<\tau-W_{1}, W_{1}<\eta \tau\right) \\
& =\int_{0}^{\eta \tau} \operatorname{Pr}\left(Y<\tau-w_{1}\right) f_{W_{1}}\left(w_{1}\right) d w_{1} \\
& =\int_{0}^{\eta \tau} F_{Y}\left(\tau-w_{1}\right) f_{W_{1}}\left(w_{1}\right) d w_{1},
\end{aligned}
$$

where recognizing that $Y$ and $W_{1}$ are exponential random variables, after the appropriate substitutions and mathematical manipulations, $T_{1}$ is obtained as in (7). In order to derive $T_{2}$, we begin by defining the variable $A \triangleq X /(U+1)$ with CDF and PDF given, respectively, by

$$
\begin{aligned}
F_{A}(a) & =\operatorname{Pr}\left(\frac{X}{U+1}<a\right) \\
& =\int_{0}^{\infty} F_{X}(a(u+1)) f_{U}(u) d u \\
& =1-\frac{e^{-\frac{a}{\gamma_{1}} \bar{\gamma}_{1}}}{\bar{\gamma}_{1}+a \bar{\gamma}_{R R}} . \\
f_{A}(a) & =e^{-\frac{a}{\gamma_{1}} \frac{\bar{\gamma}_{1}+\left(a+\bar{\gamma}_{1}\right) \bar{\gamma}_{R R}}{\left(\bar{\gamma}_{1}+a \bar{\gamma}_{R R}\right)^{2}} .}
\end{aligned}
$$

Hence, $T_{2}$ can be expressed as

$$
\begin{aligned}
T_{2}= & \operatorname{Pr}\left(\tau-W_{1}<Y<(\tau+1)^{2}-W_{1}-1, W_{1}<\eta \tau,\right. \\
& \left.\frac{A Y}{A+Y+1}<\tau-W_{1}\right) \\
= & \int_{0}^{\eta \tau} \int_{\tau-w_{1}}^{(\tau+1)^{2}-w_{1}-1} F_{A}\left[\frac{\left(\tau-w_{1}\right)(y+1)}{y-\left(\tau-w_{1}\right)}\right] f_{Y}(y) f_{W_{1}}\left(w_{1}\right) d y d w_{1} .
\end{aligned}
$$

However, in this form, the calculation of $T_{2}$ is somehow intractable and does not lead to a closed-form expression. Instead, we resort to the well-known relationship $A Y /(A+Y+1)<\min \{A, Y\}^{7}$ to find a tight approximation for $T_{2}$, which leads to

$$
\begin{aligned}
T_{2} \approx & \int_{0}^{\eta \tau} \operatorname{Pr}\left(\tau-w_{1}<Y<(\tau+1)^{2}-w_{1}-1,\right. \\
& \left.\min \{A, Y\}<\tau-w_{1}\right) f_{W_{1}}\left(w_{1}\right) d w_{1} \\
& \stackrel{(b)}{=} \int_{0}^{\eta \tau} \int_{\tau-w_{1}}^{(\tau+1)^{2}-w_{1}-1} \int_{0}^{\tau-w_{1}} f_{A}(a) f_{Y}(y) f_{W_{1}}\left(w_{1}\right) d a d y d w_{1} .
\end{aligned}
$$

where (b) is obtained by considering that the $\operatorname{CDF}$ of $\Lambda \triangleq$ $\min \{A, Y\}$ is equal to $F_{A}(\lambda)+F_{Y}(\lambda)-F_{A}(\lambda) F_{Y}(\lambda)$, and

\footnotetext{
${ }^{7}$ The approximation of the harmonic mean by the minimum leads to a tight approximation at medium-to-high SNR, which has been largely used in the literature to handle similar problems in which exact calculations prove very intricate or infeasible, so that bound or approximate calculations become necessary (see, for instance, [31], [38, eq. (29)], [39], and references therein).
} 


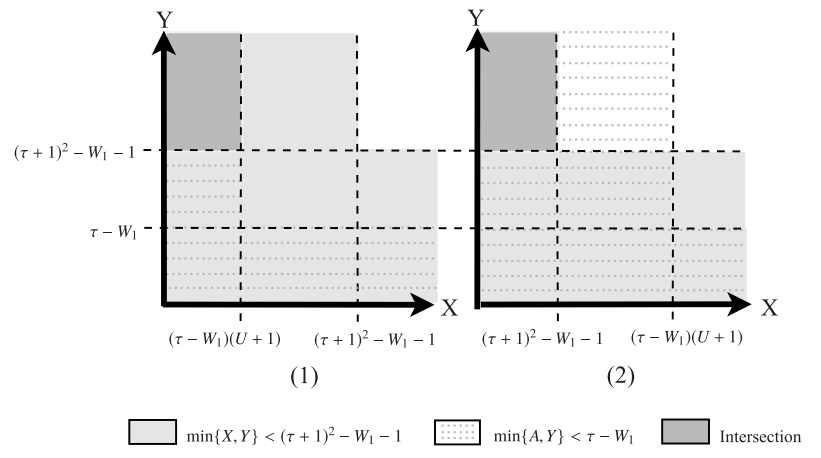

Fig. 9. Integration regions for the approximation of $T_{3}$.

that the only possible integration region for the variable $A$ is $0<A<\tau-W_{1}$, since $Y>\tau-W_{1}$. Then, with the respective substitutions, and the help of [32, eqs. (3.352-1), (3.353-1), and (3.353-3)], after some mathematical manipulations, the approximation for $T_{2}$ can be attained as in (8). Finally, $T_{3}$ can be also approximated by using the same artifice as for $T_{2}$, and it can be expressed as

$$
\begin{aligned}
T_{3}= & \operatorname{Pr}\left(W_{1}<\eta \tau, Y>(\tau+1)^{2}-W_{1}-1,\right. \\
& \left.\frac{X Y}{X+Y+1}<(\tau+1)^{2}-W_{1}-1, \frac{A Y}{A+Y+1}<\tau-W_{1}\right) \\
\approx \operatorname{Pr}( & W_{1}<\eta \tau, Y>(\tau+1)^{2}-W_{1}-1, \\
& \left.\min \{X, Y\}<(\tau+1)^{2}-W_{1}-1, \min \{A, Y\}<\tau-W_{1}\right) .
\end{aligned}
$$

This probability can be split into two cases: (i) $U<$ $\tau(\tau+1) /\left(\tau-W_{1}\right)$ and (ii) $U>\tau(\tau+1) /\left(\tau-W_{1}\right)$, whose corresponding integration regions are depicted in Figs. 9-a and 9-b (intersection regions). Then, we can calculate an approximate expression for $T_{3}$ as

$$
\begin{aligned}
T_{3} \approx & \int_{0}^{\eta \tau} \int_{(\tau+1)^{2}-w_{1}-1}^{\infty}\left[\int_{0}^{\frac{\tau(\tau+1)}{\tau-w_{1}}} \int_{0}^{\left(\tau-w_{1}\right)(u+1)} f_{X}(x) f_{U}(u) d x d u\right. \\
& \left.+\int_{\frac{\tau(\tau+1)}{\tau-w_{1}}}^{\infty} \int_{0}^{(\tau+1)^{2}-w_{1}-1} f_{X}(x) f_{U}(u) d x d u\right] \\
& \times f_{Y}(y) f_{W_{1}}\left(w_{1}\right) d y d w_{1} .
\end{aligned}
$$

After some mathematical manipulations similar to the case of $T_{2}$, an approximate expression for $T_{3}$ is finally given as in (9).

\section{APPENDIX B \\ Asymptotic Calculation for the OUtage PROBABILITY OF THE ATMS}

In the high SNR regime, as the transmit system SNR $\bar{\gamma}$ increases, note that $1 / \bar{\gamma}_{0}, 1 / \bar{\gamma}_{1}, 1 / \bar{\gamma}_{2}$, and $1 / \bar{\gamma}_{R R}$ decreases. Then, when $\bar{\gamma}$ goes to $\infty, 1 / \bar{\gamma}_{0}, 1 / \bar{\gamma}_{1}, 1 / \bar{\gamma}_{2}$, and $1 / \bar{\gamma}_{R R}$ go to zero. By considering the Maclaurin series expansion of the exponential function [32, eq. (0.318.2)], and by omitting its higher-order terms, it follows that $e^{-b} \simeq 1-b$ as $b$ goes to zero. Using this, $F_{A}(a)$ in (34) can be asymptotically expressed as

$$
F_{A}(a)^{\infty}=\frac{a\left(1+\bar{\gamma}_{R R}\right)}{\bar{\gamma}_{1}+a \bar{\gamma}_{R R}}
$$

Taking this into consideration, all the PDFs and CDFs in (33), (36), and (38) can be asymptotically expressed. Thus, by solving the corresponding integrals and after a process of simplification of the higher-order terms, the expression in (11) can be finally attained.

\section{APPENDIX C \\ Approximate Calculation for the Probability of SELECTION OF THE DifFERENT SCHEMES}

In the following, we derive the probabilities of each transmission mode being selected in the ATMS.

Direct Transmission Mode: Regarding (4) and (5), the probability to select DT can be formulated as

$\mathcal{P}_{\mathrm{DT}}$

$$
\begin{aligned}
& =\operatorname{Pr}\left(I_{\mathrm{DT}}>I_{\mathrm{HD}-\mathrm{MRC}}, I_{\mathrm{DT}}>I_{\mathrm{FD}-\mathrm{MRC}}\right) \\
& =\operatorname{Pr}\left(W_{0}>\sqrt{1+\frac{X Y}{X+Y+1}+W_{1}}-1, W_{0}>\frac{A Y}{A+Y+1}+W_{1}\right) \\
& =\operatorname{Pr}\left(\frac{X Y}{X+Y+1}<\left(W_{0}+1\right)^{2}-\eta W_{0}-1, \frac{A Y}{A+Y+1}<W_{0}-\eta W_{0}\right) \\
& \stackrel{(c)}{=} \operatorname{Pr}\left(X<\frac{\left[\left(W_{0}+1\right)^{2}-\eta W_{0}-1\right](Y+1)}{Y-\left[\left(W_{0}+1\right)^{2}-\eta W_{0}-1\right]}, X<\frac{\left(W_{0}-\eta W_{0}\right)(Y+1)(U+1)}{Y-\left(W_{0}-\eta W_{0}\right)}\right) .
\end{aligned}
$$

where (c) is obtained by isolating the random variable $X$ in both events. Then, by applying a rationale similar to that used to find the outage probability in Section III and considering that $\left(W_{0}+1\right)^{2}-\eta W_{0}-1>W_{0}-\eta W_{0}$, we split the analysis of the probability above into three regions for the random variable $Y$, namely (i) $Y<(1-\eta) W_{0}$, (ii) $(1-\eta) W_{0}<$ $Y<\left(W_{0}+1\right)^{2}-\eta W_{0}-1$, and (iii) $Y>\left(W_{0}+1\right)^{2}-\eta W_{0}-1$, yielding

$$
\mathcal{P}_{\mathrm{DT}}=\mathcal{P}_{\mathrm{DT}}^{1}+\mathcal{P}_{\mathrm{DT}}^{2}+\mathcal{P}_{\mathrm{DT}}^{3},
$$

where $\mathcal{P}_{\mathrm{DT}}^{1}$ is given by

$$
\begin{aligned}
\mathcal{P}_{\mathrm{DT}}^{1} & =\operatorname{Pr}\left(Y<(1-\eta) W_{0}\right) \\
& =\int_{0}^{\infty} F_{Y}\left[(1-\eta) w_{0}\right] f_{W_{0}}\left(w_{0}\right) d w_{0} .
\end{aligned}
$$

The solution of this integral leads to the expression in (20). For the calculation of $\mathcal{P}_{\mathrm{DT}}^{2}$ and $\mathcal{P}_{\mathrm{DT}}^{3}$ we make use of the approximation of the harmonic mean by the minimum, that is $\frac{A Y}{A+Y+1}<\min \{A, Y\}$ or $\frac{X Y}{X+Y+1}<\min \{X, Y\}$ as required. In this sense, an approximate expression for $\mathcal{P}_{\mathrm{DT}}^{2}$ can be found as follows:

$$
\begin{aligned}
& \mathcal{P}_{\mathrm{DT}}^{2} \approx \operatorname{Pr}\left((1-\eta) W_{0}<Y<\left(W_{0}+1\right)^{2}-\eta W_{0}-1,\right. \\
&\left.\min \{A, Y\}<(1-\eta) W_{0}\right) \\
&=\int_{0}^{\infty} \int_{0}^{\infty} \int_{0}^{(1-\eta) w_{0}(u+1)} \int_{(1-\eta) w_{0}}^{\left(w_{0}+1\right)^{2}-\eta w_{0}-1} f_{Y}(y) f_{X}(x) f_{U}(u) \\
& \quad \times f_{W_{0}}\left(w_{0}\right) d y d x d u d w_{0} .
\end{aligned}
$$


By following a similar process as for the case of (39), we arrive at the expression in (21). On the other hand, an approximation for $\mathcal{P}_{\mathrm{DT}}^{3}$ can be attained by considering two regions for the random variable $U$, analogously to the case shown in Fig. 9: (i) $U<\left(W_{0}+1\right) /(1-\eta)$ and (ii) $U>$ $\left(W_{0}+1\right) /(1-\eta)$, which lead to the following formulation:

$$
\begin{aligned}
& \mathcal{P}_{\mathrm{DT}}^{3} \\
& \approx \operatorname{Pr}\left(Y>\left(W_{0}+1\right)^{2}-\eta W_{0}-1, \min \{A, Y\}<(1-\eta) W_{0},\right. \\
& \left.\quad \min \{X, Y\}<\left(W_{0}+1\right)^{2}-\eta W_{0}-1\right) \\
& =\int_{0}^{\infty} \int_{0}^{\frac{w_{0}+1}{1-\eta}} \int_{0}^{(1-\eta) w_{0}(u+1)} \int_{\left(w_{0}+1\right)^{2}-\eta w_{0}-1}^{\infty} f_{Y}(y) f_{X}(x) f_{U}(u) \\
& \quad \times f_{W_{0}}\left(w_{0}\right) d y d x d u d w_{0} \\
& \quad+\int_{0}^{\infty} \int_{\frac{w_{0}+1}{1-\eta}}^{\infty} \int_{0}^{\left(w_{0}+1\right)^{2}-\eta w_{0}-1} \int_{\left(w_{0}+1\right)^{2}-\eta w_{0}-1}^{\infty} f_{Y}(y) f_{X}(x) f_{U}(u) \\
& \quad \times f_{W_{0}}\left(w_{0}\right) d y d x d u d w_{0} .
\end{aligned}
$$

The above formula can be solved in single-fold integral form, finally arriving at the expression in (22).

Full-Duplex MRC Transmission Mode: Analogously to the DT mode, we can formulate the probability of selection for the probability of the FD-MRC mode as

$$
\begin{aligned}
\mathcal{P}_{\mathrm{FD}-\mathrm{MRC}}= & \operatorname{Pr}\left(I_{\mathrm{FD}-\mathrm{MRC}}>I_{\mathrm{DT}}, I_{\mathrm{FD}-\mathrm{MRC}}>I_{\mathrm{HD}-\mathrm{MRC}}\right) \\
=\operatorname{Pr} & \left(\frac{A Y}{A+Y+1}+W_{1}>W_{0},\right. \\
& \left.\frac{A Y}{A+Y+1}+W_{1}>\sqrt{1+\frac{X Y}{X+Y+1}+W_{1}}-1\right) .
\end{aligned}
$$

This probability can be rewritten as

$$
\begin{aligned}
& \mathcal{P}_{\mathrm{FD}-\mathrm{MRC}}=\operatorname{Pr}\left(\frac{A Y}{A+Y+1}+W_{1}+1>W_{0}+1,\right. \\
&\left.\frac{A Y}{A+Y+1}+W_{1}+1>\sqrt{1+\frac{X Y}{X+Y+1}+W_{1}}\right) .
\end{aligned}
$$

In order to simplify the analysis, we can split this probability into two terms, by considering the following cases: (i) $\frac{X Y}{X+Y+1}<\left(W_{0}+1\right)^{2}-\eta W_{0}-1$ and (ii) $\frac{X Y}{X+Y+1}>\left(W_{0}+1\right)^{2}-$ $\eta W_{0}-1$. For the first case we have that

$$
\begin{aligned}
& \mathcal{P}_{\mathrm{FD}-\mathrm{MRC}}^{A}=\operatorname{Pr}\left(\frac{A Y}{A+Y+1}+\right. W_{1}+1>W_{0}+1, \\
&\left.\frac{X Y}{X+Y+1}<\left(W_{0}+1\right)^{2}-\eta W_{0}-1\right) .
\end{aligned}
$$

In this case, a procedure similar to that applied to analyze the probability of the DT mode is considered, with the same regions for the random variable $Y$, yielding three corresponding terms for $\mathcal{P}_{\mathrm{FD}-\mathrm{MRC}}^{A}$. The first region, i.e., $Y<$ $(1-\eta) W_{0}$, produces an event with zero probability, thus $\mathcal{P}_{\mathrm{FD}-\mathrm{MRC}}^{A 1}=0$. For the second region, we can again use the approximation of the harmonic mean by the minimum, leading to

$$
\begin{aligned}
\mathcal{P}_{\mathrm{FD}-\mathrm{MRC}}^{A 2} & \operatorname{Pr}\left(\min \{A, Y\}>(1-\eta) W_{0},\right. \\
\approx & \left.(1-\eta) W_{0}<Y<\left(W_{0}+1\right)^{2}-\eta W_{0}-1\right) \\
= & \int_{0}^{\infty} \int_{0}^{\infty} \int_{(1-\eta) w_{0}(u+1)}^{\infty} \int_{(1-\eta) w_{0}}^{\left(w_{0}+1\right)^{2}-\eta w_{0}-1} f_{Y}(y) f_{X}(x) f_{U}(u) \\
& \times f_{W_{0}}\left(w_{0}\right) d y d x d u d w_{0} .
\end{aligned}
$$

After some mathematical manipulations, we arrive at the expression in (25). Finally, the third region can also be approximated by the minimum, then yielding two subterms according to the cases (i) $U>\frac{W_{0}+1}{1-\eta}$ and (ii) $U<\frac{W_{0}+1}{1-\eta}$. The case (i) also leads to zero probability, and the case (ii) leads to the following formulation:

$$
\begin{aligned}
& \mathcal{P}_{\mathrm{FD}-\mathrm{MRC}}^{A 3} \\
& \approx \operatorname{Pr}\left(\min \{X, Y\}<\left(W_{0}+1\right)^{2}-\eta W_{0}-1,\right. \\
& \left.\quad \min \{A, Y\}>(1-\eta) W_{0}, Y>\left(W_{0}+1\right)^{2}-\eta W_{0}-1\right) \\
& =\int_{0}^{\infty} \int_{0}^{\frac{w_{0}+1}{1-\eta}} \int_{(1-\eta) w_{0}(u+1)} \int_{\left(w_{0}+1\right)^{2}-\eta w_{0}-1}^{\infty} f_{Y}(y) f_{X}(x) f_{U}(u) \\
& \quad \times f_{W_{0}}\left(w_{0}\right) d y d x d u d w_{0} .
\end{aligned}
$$

A single-fold integral-form solution to this expression can be found as in (26).

Now, for case (b), we have the following formulation:

$$
\begin{aligned}
\mathcal{P}_{\mathrm{FD}-\mathrm{MRC}}^{B}= & \operatorname{Pr}\left(\frac{A Y}{A+Y+1}+W_{1}+1>\sqrt{1+\frac{X Y}{X+Y+1}+W_{1}},\right. \\
& \left.\frac{X Y}{X+Y+1}>\left(W_{0}+1\right)^{2}-\eta W_{0}-1\right) .
\end{aligned}
$$

In this form, the evaluation of this probability is intractable. Then, we propose an approximation for very low values of $U$. Indeed, the values assumed herein are quite conservative compared to the levels reported by the state-of-the-art interference cancellation (combining antenna isolation, analog and digital cancellation), which are close to the noise floor, (see, for instance [40]), so that $\frac{A Y}{A+Y+1} \approx \frac{X Y}{X+Y+1}$. Then, we can rewrite (51) as

$$
\begin{aligned}
\mathcal{P}_{\mathrm{FD}-\mathrm{MRC}}^{B} \approx \operatorname{Pr} & \left(\frac{X Y}{X+Y+1}+W_{1}+1>1,\right. \\
& \left.\frac{X Y}{X+Y+1}+W_{1}+1>\left(W_{0}+1\right)^{2}\right) .
\end{aligned}
$$

Here, the term $\left(W_{0}+1\right)^{2}$ is always greater than 1. Again, by using the approximation for the harmonic mean, this probability can be reduced to

$$
\mathcal{P}_{\mathrm{FD}-\mathrm{MRC}}^{B} \approx \operatorname{Pr}\left(\frac{X Y}{X+Y+1}>\left(W_{0}+1\right)^{2}-\eta W_{0}-1\right) .
$$

Thus, for the case $Y<\left(W_{0}+1\right)^{2}-\eta W_{0}-1$, this probability equals 0 . Otherwise, for $Y>\left(W_{0}+1\right)^{2}-\eta W_{0}-1$, we 
have that

$$
\begin{aligned}
\mathcal{P}_{\mathrm{FD} \text {-MRC }}^{B} \approx & \int_{0}^{\infty} \int_{\left(w_{0}+1\right)^{2}-\eta w_{0}-1}^{\infty} \int_{\left(w_{0}+1\right)^{2}-\eta w_{0}-1}^{\infty} f_{Y}(y) f_{X}(x) \\
& \times f_{W_{0}}\left(w_{0}\right) d y d x d w_{0} .
\end{aligned}
$$

After simplifying this multi-fold integral, we obtain the expression in (27). Finally, the probability of the FD-MRC transmission mode is approximately given by $\mathcal{P}_{\mathrm{FD}-\mathrm{MRC}} \approx$ $\mathcal{P}_{\mathrm{FD}-\mathrm{MRC}}^{A 2}+\mathcal{P}_{\mathrm{FD}-\mathrm{MRC}}^{A 3}+\mathcal{P}_{\mathrm{FD}-\mathrm{MRC}}^{B}$

Having obtained the selection probabilities for DT and FD-MRC, the selection probability for HD-MRC can be calculated as in (28).

\section{REFERENCES}

[1] T. Riihonen, S. Werner, R. Wichman, and Z. B. Eduardo, "On the feasibility of full-duplex relaying in the presence of loop interference," in Proc. IEEE 10th Workshop Signal Process. Adv. Wireless Commun. SPAWC, Perugia, Italy, Jun. 2009, pp. 275-279.

[2] T. Riihonen, S. Werner, and R. Wichman, "Comparison of full-duplex and half-duplex modes with a fixed amplify-and-forward relay," in Proc. IEEE WCNC, Budapest, Hungary, Apr. 2009, pp. 1-5.

[3] Y. Y. Kang and J. H. Cho, "Capacity of MIMO wireless channel with full-duplex amplify-and-forward relay," in Proc. IEEE 20th Int Symp. Pers. Indoor Mobile Radio Commun., Tokyo, Japan, Sep. 2009, pp. 117-121.

[4] B. Chun and Y. H. Lee, "A spatial self-interference nullification method for full duplex amplify-and-forward MIMO relays," in Proc. IEEE Wireless Commun. Netw. Conf. (WCNC), Sydney, NSW, Australia, Apr. 2010, pp. 1-6.

[5] T. Riihonen, S. Werner, and R. Wichman, "Residual self-interference in full-duplex MIMO relays after null-space projection and cancellation," in Proc. 44th Conf. Signals, Syst., Comput. (ASILOMAR), Pacific Grove, CA, USA, Nov. 2010, pp. 653-657.

[6] M. Duarte, C. Dick, and A. Sabharwal, "Experiment-driven characterization of full-duplex wireless systems," IEEE Trans. Wireless Commun. vol. 11 , no. 12, pp. 4296-4307, Dec. 2012

[7] J. I. Choi, M. Jain, K. Srinivasan, P. Levis, and S. Katti, "Achieving single channel, full duplex wireless communication," in Proc. 16th MobCom, New York, NY, USA, 2010, pp. 1-12

[8] S. Hong et al., "Applications of self-interference cancellation in $5 \mathrm{G}$ and beyond," IEEE Commun. Mag., vol. 52, no. 2, pp. 114-121, Feb. 2014.

[9] H. Ju, S. Lee, K. Kwak, E. Oh, and D. Hong, "A new duplex without loss of data rate and utilizing selection diversity," in Proc. IEEE Veh. Technol. Conf. Singapore, May 2008, pp. 1519-1523.

[10] J. Lee and T. Q. S. Quek, "Hybrid full-/half-duplex system analysis in heterogeneous wireless networks," IEEE Trans. Wireless Commun., vol. 14, no. 5, pp. 2883-2895, May 2015.

[11] H. Tabassum, A. H. Sakr, and E. Hossain, "Massive MIMO-enabled wireless backhauls for full-duplex small cells," in Proc. IEEE Global Commun. Conf. (GLOBECOM), San Diego, CA, USA, Dec. 2015, pp. 1-6.

[12] A. Sabharwal, P. Schniter, D. Guo, D. W. Bliss, S. Rangarajan, and R. Wichman, "In-band full-duplex wireless: Challenges and opportunities," IEEE J. Sel. Areas Commun., vol. 32, no. 9, pp. 1637-1652, Sep. 2014.

[13] I. Krikidis, H. A. Suraweera, P. J. Smith, and C. Yuen, "Full-duplex relay selection for amplify-and-forward cooperative networks," IEEE Trans. Wireless Commun., vol. 11, no. 12, pp. 4381-4393, Dec. 2012.

[14] M. Ghoraishi, D. Liang, G. Chen, K. Rikkinen, and V. Tapio. (2015). Enhanced Solutions for Full-Duplex Transceivers and Systems DUPLO Deliverable D3.2. [Online]. Available: http://cordis.europa.eu/docs/projects/cnect/9/316369/080/deliverables/ 001-316369DUPLOD32renditionDownload.pdf

[15] T. Kwon, S. Lim, S. Choi, and D. Hong, "Optimal duplex mode for DF relay in terms of the outage probability," IEEE Trans. Veh. Technol., vol. 59, no. 7, pp. 3628-3634, Sep. 2010.

[16] H. Alves, D. B. da Costa, R. D. Souza, and M. Latva-aho, "Performance of Block-Markov full duplex relaying with self interference in Nakagami- $m$ fading," IEEE Wireless Commun. Lett., vol. 2, no. 3 , pp. 311-314, Jun. 2013 .
[17] Q. Wang, Y. Dong, X. Xu, and X. Tao, "Outage probability of full-duplex AF relaying with processing delay and residual self-interference," IEEE Commun. Lett., vol. 19, no. 5, pp. 783-786, May 2015.

[18] T. Riihonen, S. Werner, and R. Wichman, "Optimized gain control for single-frequency relaying with loop interference," IEEE Trans. Wireless Commun., vol. 8, no. 6, pp. 2801-2806, Jun. 2009.

[19] L. Jimenez Rodriguez, N. H. Tran, and T. Le-Ngoc, "Optimal power allocation and capacity of full-duplex AF relaying under residual selfinterference," IEEE Wireless Commun. Lett., vol. 3, no. 2, pp. 233-236, Apr. 2014.

[20] T. Riihonen, S. Werner, and R. Wichman, "Hybrid full-duplex/halfduplex relaying with transmit power allocation," IEEE Trans. Wireless Commun., vol. 10, no. 9, pp. 3074-3085, Nov. 2011.

[21] K. Yamamoto, K. Haneda, H. Murata, and S. Yoshida, "Optimal transmission scheduling for a hybrid of full- and half-duplex relaying," IEEE Commun. Lett., vol. 15, no. 3, pp. 305-307, Mar. 2011.

[22] D. W. Kwan Ng, E. S. Lo, and R. Schober , "Dynamic resource allocation in MIMO-OFDMA systems with full-duplex and hybrid relaying," IEEE Trans. Commun., vol. 60, no. 5, pp. 1291-1304, May 2012.

[23] T. M. Kim and A. Paulraj, "Outage probability of amplify-and-forward cooperation with full duplex relay," in Proc. IEEE Wireless Commun. Netw. Conf. (WCNC), Shanghai, China, Apr. 2012, pp. 75-79.

[24] M. G. Khafagy, A. Ismail, M.-S. Alouini, and S. Aïssa, "Efficient cooperative protocols for full-duplex relaying over Nakagami- $m$ fading channels," IEEE Trans. Wireless Commun., vol. 14, no. 6, pp. 3456-3470, Jun. 2015.

[25] L. Jiménez Rodríguez, N. H. Tran, and T. Le-Ngoc, "Performance of full-duplex AF relaying in the presence of residual self-interference," IEEE J. Sel. Areas Commun., vol. 32, no. 9, pp. 1752-1764, Sep. 2014.

[26] D. P. M. Osorio, E. E. B. Olivo, H. Alves, J. C. S. S. Filho, and M. Latva-aho, "Exploiting the direct link in full-duplex amplify-andforward relaying networks," IEEE Signal Process. Lett., vol. 22, no. 10, pp. 1766-1770, May 2015.

[27] L. Li, L. J. Cimini, and X.-G. Xia, "Impact of direct link on outage of cooperative full-duplex relaying," in Proc. 49th Annu. Conf. Inf. Sci. Syst. (CISS), Baltimore, MD, USA, Mar. 2015, pp. 1-6.

[28] M. E. Knox, "Single antenna full duplex communications using a common carrier," in Proc. IEEE 13th Annu. Wireless Microw. Technol. Conf. (WAMICON), Cocoa Beach, FL, USA, Apr. 2012, pp. 1-6.

[29] J. N. Laneman, D. N. C. Tse, and G. W. Wornell, "Cooperative diversity in wireless networks: Efficient protocols and outage behavior," IEEE Trans. Inf. Theory, vol. 50, no. 12, pp. 3062-3080, Dec. 2004.

[30] M. Liu, J. Zhang, Y. Zhang, and Y. Liu, "A channel estimation scheme for amplify-and-forward OFDM relay networks," in Proc. IEEE 70th Veh. Technol. Conf. Fall, Anchorage, AK, USA, Sep. 2009, pp. 1-5.

[31] H. Ding, J. Ge, D. B. da Costa, and Z. Jiang, "Link selection schemes for selection relaying systems with transmit beamforming: New and efficient proposals from a distributed concept," IEEE Trans. Veh. Technol., vol. 61 , no. 2, pp. 533-552, Feb. 2012

[32] I. Gradshteyn and I. Ryzhik, Table of Integrals, Series and Products, 7th ed. Washington, DC, USA: Academic Press, 2007.

[33] H. Alves, R. D. Souza, and G. Fraidenraich, "Outage, throughput and energy efficiency analysis of some half and full duplex cooperative relaying schemes," Trans. Emerg. Telecommun. Technol., vol. 25, no. 11, pp. 1114-1125, Nov. 2014

[34] S. Cui, A. J. Goldsmith, and A. Bahai, "Energy-constrained modulation optimization," IEEE Trans. Wireless Commun., vol. 4, no. 5, pp. 2349-2360, Sep. 2005.

[35] N. Yang, M. Elkashlan, and J. Yuan, "Outage probability of multiuser relay networks in Nakagami- $m$ fading channels," IEEE Trans. Veh. Technol., vol. 59, no. 5, pp. 2120-2132, Jun. 2010.

[36] Jun. 2015. Wolfram: NArgMin function. [Online]. Available: https://reference.wolfram.com/language/ref/NArgMin.html

[37] M. Abramowitz and I. Stegun, Handbook of Mathematical Functions with Formulas, Graphs, and Mathematical Tables. New York, NY, USA: Dover, 1972.

[38] M. Xia and S. Aïssa, "Cooperative AF relaying in spectrum-sharing systems: Outage probability analysis under co-channel interferences and relay selection," IEEE Trans. Commun., vol. 60, no. 11, pp. 3252-3262, Nov. 2012.

[39] P. A. Anghel and M. Kaveh, "Exact symbol error probability of a cooperative network in a Rayleigh-fading environment," IEEE Trans. Wireless Commun., vol. 3, no. 5, pp. 1416-1421, Sep. 2004.

[40] B. Debaillie et al., "Analog/RF solutions enabling compact full-duplex radios," IEEE J. Sel. Areas Commun., vol. 32, no. 9, pp. 1662-1673, Sep. 2014 


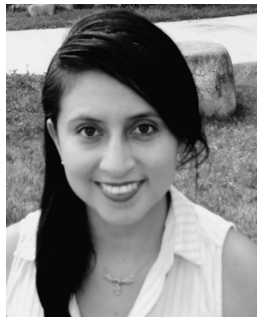

Diana Pamela Moya Osorio (M'16) was born in Quito, Ecuador. She received the B.Sc. degree in electrical and telecommunications engineering from Armed Forces University-ESPE, Sangolquí, Ecuador, in 2008, and the M.Sc. and D.Sc. degrees in electrical engineering from the University of Campinas, Campinas, SP, Brazil, in 2011 and 2015 , respectively. She is currently an Assistant Professor with the Department of Electrical Engineering, Federal University of São Carlos, São Carlos, SP, Brazil. Her research interests include wireless communications in general, cooperative relaying networks, cognitive radio systems, physical layer security, and technologies for $5 \mathrm{G}$ networks.

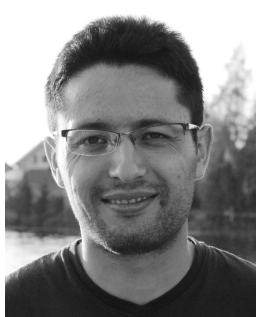

Edgar Eduardo Benítez Olivo (M'16) received the B.Sc. degree in electrical and telecommunications engineering from Armed Forces University-ESPE, Sangolquí, Ecuador, in 2008, and the M.Sc. and Ph.D. degrees in electrical engineering from the University of Campinas, Campinas, SP, Brazil, in 2011 and 2015, respectively. In 2014, he held a visiting researcher position with the Centre for Wireless Communications, University of Oulu, Finland. He is currently an Assistant Professor with São Paulo State University (UNESP), São Jõao da Boa Vista, SP, Brazil. His research interests lie in the area of wireless communications, with a current focus on emerging technologies towards $5 \mathrm{G}$ wireless networks.

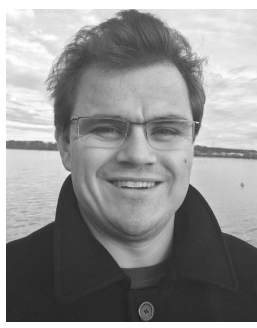

Hirley Alves (S'11-M'16) received the B.Sc., M.Sc., and D.Sc. degrees in electrical engineering from the Federal University of Technology-Paraná, Brazil, in 2010, 2011, and 2015, respectively. Hirley has jointly graduated from University of Oulu, and received his D.Sc. in 2015. He is a Researcher with the Centre for Wireless Communications, Oulu. His current research focuses on ultrareliable communication on machine type networks, performance analysis of full-duplex relaying networks, physical layer security, 5G networks, and smart grids.

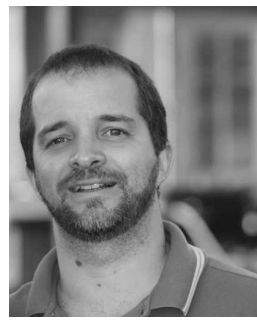

José Cândido Silveira Santos Filho (M'09) received the B.Sc. (Hons.), M.Sc., and Ph.D. degrees from the School of Electrical and Computer Engineering (FEEC), University of Campinas (UNICAMP), Campinas, SP, Brazil, in 2001, 2003, and 2006, respectively, all in electrical engineering. From 2006 to early 2009, he was a Post-Doctoral Fellow with the Wireless Technology Laboratory, FEEC-UNICAMP. He is currently an Assistant Professor with FEEC-UNICAMP. Since 2011, he has been a regular consultant for Bradar Indústria S.A., a branch of Embraer Defense and Security, in the development of innovative radar techniques. He has authored or co-authored over 70 technical papers, about half of which in international journals. His Ph.D. thesis was awarded an Honorary Mention by the Brazilian Ministry of Education (CAPES) in the 2007 CAPES Thesis Contest. He has served as a Reviewer for many journals and conferences. His research areas include wireless communications and radar systems.

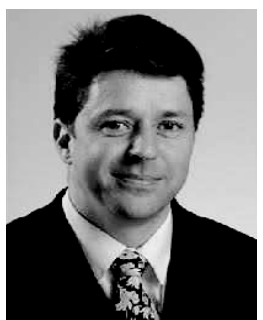

Matti Latva-aho (S'96-M'98-SM'06) was born in Kuivaniemi, Finland, in 1968. He received the M.Sc., Lic.Tech., and Dr. Tech (Hons.) degrees in electrical engineering from the University of Oulu, Finland, in 1992, 1996, and 1998, respectively. From 1992 to 1993, he was a Research Engineer with Nokia Mobile Phones, Oulu, Finland. From 1994 to 1998 , he was a Research Scientist with the Telecommunication Laboratory, Centre for Wireless Communications, University of Oulu. He was the Director of the Centre for Wireless Communications, University of Oulu from 1998 to 2006. He is currently the Chair of the Department of Communications Engineering and Professor of Digital Transmission Techniques, University of Oulu. 